Draft Version OCtOBer 29, 2018

Preprint typeset using $\mathrm{LAT}_{\mathrm{E}} \mathrm{X}$ style emulateapj v. 08/22/09

\title{
PHOTOMETRIC PROPERTIES OF THE MOST MASSIVE HIGH-REDSHIFT GALAXIES
}

\author{
Brant Robertson ${ }^{1,2,4,5}$, Yuexing Li $^{3}$, Thomas J. Cox ${ }^{3}$, Lars Hernquist ${ }^{3}$ \& Philip F. Hopkins ${ }^{3}$ \\ Draft version October 29, 2018
}

\begin{abstract}
We calculate the observable properties of the most massive high-redshift galaxies in the hierarchical formation scenario where stellar spheroid and supermassive black hole growth are fueled by gasrich mergers. Combining high-resolution hydrodynamical simulations of the hierarchical formation of a $z \sim 6$ quasar, stellar population synthesis models, template AGN spectra, prescriptions for interstellar and intergalactic absorption, and the response of modern telescopes, the photometric evolution of galaxies destined to host $z \sim 6$ quasars are modeled at redshifts $z \sim 4-14$. These massive galaxies, with enormous stellar masses of $M_{\star} \sim 10^{11.5}-10^{12} M_{\odot}$ and star formation rates of $\mathrm{SFR} \sim 10^{3}-10^{4} M_{\odot} \mathrm{yr}^{-1}$ at $z \gtrsim 7$, satisfy a variety of photometric selection criteria based on Lymanbreak techniques including $V$-band dropouts at $z \gtrsim 5, i$-band dropouts at $z \gtrsim 6$, and $z$-band dropouts at $z \gtrsim 7$. The observability of the most massive high-redshift galaxies is assessed and compared with a wide range of existing and proposed photometric surveys including SDSS, GOODS/HUDF, NOAO WDFS, UKIDSS, the IRAC Shallow Survey, Ultra-VISTA, DUNE, Pan-STARRS, LSST, and SNAP. Massive stellar spheroids descended from $z \sim 6$ quasars will likely be detected at $z \sim 4$ by existing surveys, but owing to their low number densities the discovery of quasar progenitor galaxies at $z>7$ will likely require future surveys of large portions of the sky $(\gtrsim 0.5 \%)$ at wavelengths $\lambda \gtrsim 1 \mu \mathrm{m}$. The detection of rare, star-bursting, massive galaxies at redshifts $z \gtrsim 6$ would provide support for the hierarchical formation of the earliest quasars and characterize the primitive star-formation histories of the most luminous elliptical galaxies.
\end{abstract}

Subject headings: galaxies: formation - galaxies: evolution

\section{INTRODUCTION}

Supermassive black holes (SMBHs) serve as the relativistic engines of quasars (Lynden-Bell 1969) and the existence of quasars at $z \sim 6$ suggests the rapid formation of SMBHs with masses $M_{\mathrm{BH}} \sim 10^{9} M_{\odot}$ (e.g., Fan et al. 2000, 2001a, 2003, 2004, 2006). Examining the consistency of rapid SMBH growth and the formation of cold dark matter (CDM) structures at highredshifts has been of long standing theoretical interest (e.g., Efstathiou \& Rees 1988), as a confluence of the deep potential well of a rare, massive dark matter halo, an abundant supply of baryons, and an efficient SMBH fueling mechanism is likely needed to grow enormous SMBHs in the available $\approx 900$ Myrs before redshift $z \sim 6$. The existence of quasars at $z \sim 6$ can be considered a posteriori evidence that our currently dark energy-dominated $(\Lambda \mathrm{CDM})$ universe can muster this confluence, but little else is known observationally about the associated formation of quasar host galaxies at such high redshifts. The focus of this paper is to assist in the detection and characterization of massive galaxies destined to host $z \sim 6$ quasars by calculating their photometric evolution in the scenario where $\Lambda$ CDM structure formation fuels SMBHs and grows stellar spheroids through gas-rich mergers.

\footnotetext{
${ }^{1}$ Kavli Institute for Cosmological Physics, and Department of Astronomy and Astrophysics, University of Chicago, 933 East 56th Street, Chicago, IL 60637, USA

2 Enrico Fermi Institute, 5640 South Ellis Avenue, Chicago, IL 60637, USA

${ }^{3}$ Harvard-Smithsonian Center for Astrophysics, 60 Garden St., Cambridge, MA 02138, USA

${ }_{5}^{4}$ Spitzer Fellow

${ }^{5}$ brant@kicp.uchicago.edu
}

The formation of stellar spheroids through mergers of disk galaxies has been a long-established vehicle for galaxy evolution (e.g., Toomre \& Toomre 1972; Toomre 1977). If the disk galaxies contain gas before the merger, gravitational torques can enable gas to loose angular momentum, flow efficiently to the centers of the merging systems, and result in a starburst (Larson \& Tinslev 1978; Noguchi 1988; Hernquist 1989; Barnes \& Hernquist 1991, 1996; Mihos \& Hernquist 1994, 1996). The efficient fueling of starbursts by mergers may explain ultraluminous infrared galaxies (ULIRGS, e.g. Soifer et al. 1984; Young et al. 1986; Soifer et al. 1987; Sanders \& Mirabel 1996) as an intermediate phase where the high infrared luminosities are powered by star formation before gas accretion can grow central supermassive black holes to be massive enough to become luminous quasars (Sanders et al. 1988b;a; Hernquist 1989; Sanders \& Mirabel 1996; Genzel et al. 1998). This evolutionary picture would paint starbursts, ULIRGS, and quasars as stages in a structure formation process that builds spheroid and SMBH mass contemporaneously through galaxy mergers.

The observed correlations between SMBH mass and stellar spheroid mass (the $M_{\mathrm{BH}}-M_{\text {bulge }}$ relation, e.g. Kormendy \& Richstone 1995; Magorrian et al. 1998; Marconi et al. 2003; Häring \& Rix 2004), SMBH mass and stellar velocity dispersion (the $M_{\mathrm{BH}}-\sigma$ relation, Ferrarese \& Merritt 2000; Gebhardt et al. 2000; Tremaine et al. 2002), and the black hole fundamental plane (Hopkins et al. 2007a) have been widely interpreted as evidence for the coeval or coupled formation of SMBHs and stellar spheroids (e.g. Silk \& Rees 1998; Fabian 1999; Kauffmann \& Haehnelt 
2000; Volonteri et al. 2003; Wvithe \& Loeb 2003; King 2003; Granato et al. 2004; Hopkins et al. 2006a,b). Hydrodynamical simulations of the formation of SMBHs and their host spheroids in gas-rich mergers (Di Matteo et al. 2005; Robertson et al. 2006b) that include a prescription for SMBH growth and feedback (Springel et al. 2005b) generically predict that such mergers undergo starbursts, consistent with earlier simulations that did not include SMBH modeling (e.g., Mihos \& Hernquist 1994; Barnes \& Hernquist 1996), and that the spheroid forms primarily before the growing SMBHs reach their final mass. Once the rapidly growing $\mathrm{SMBHs}$ become massive enough to drive energetic outflows, the SMBH feedback truncates star formation (Di Matteo et al. 2005; Springel et al. 2005a) and becomes visible as a luminous quasar as the outflow removes obscuring material at the galaxy center (Hopkins et al. 2005). Ultimately, the end state of this dissipative process is a spheroidal remnant that closely matches observed elliptical galaxy structural (e.g., Robertson et al. 2006a) and kinematic (e.g., Cox et al. 2006b) properties and satisfies the observed SMBH-spheroid correlations (Di Matteo et al. 2005; Robertson et al. 2006b; Hopkins et al. 2007a). The general consistency of the evolutionary sequence as detailed by these simulations with available observational data at redshifts $z \lesssim 3$ has been discussed elsewhere (e.g., Hopkins et al. 2006a, b, c).

While a developmental connection between stellar spheroids and the SMBHs they host has observational support in the nearby universe, the properties of $z \sim 6$ quasars may complicate this simple coeval picture. The Sloan Digital Sky Survey (SDSS; York et al. 2000) was used in concert with Two Micron All Sky Survey J-band imaging (2MASS; Skrutskie et al. 1997) to photometrically identify the quasar SDSS J1148+5251, spectroscopically confirmed to reside at redshift $z=6.42$ (Fan et al. 2003). Estimates of the SMBH mass from MgII and CIV line widths for this quasar are $M_{\mathrm{BH}} \sim 2-6 \times 10^{9}$ (Willott et al. 2003; Barth et al. 2003), suggesting that the SMBH is near its Eddington limit.

Radio observations of SDSS J1148+5251 infer the presence of molecular hydrogen from $\mathrm{CO}(3-2)$ emission, with $M_{\mathrm{H} 2}=2.2 \times 10^{10} M_{\odot}$ (Walter et al. 2003). The dynamical mass for the quasar host $M_{\text {dvn }} \sim 4.5 \times 10^{10} M_{\odot}$, estimated from CO line widths (Walter et al. 2003, 2004), suggests that the molecular gas and SMBH, rather than some dominant stellar component, supply the majority of the local potential within $\sim 2.5 \mathrm{kpc}$. However, radiative transfer calculations of $\mathrm{CO}$ line emission in quasars show that owing to projection effects the small observed line widths could be consistent with presence of a massive bulge in the quasar host galaxy, but such observed orientations are unlikely (Naravanan et al. 2006). The large IR luminosity $L_{\mathrm{IR}} \approx 10^{13} L_{\odot}$ (Bertoldi et al. 2003) and radio continuum imaging (Carilli et al. 2004) imply a star formation rate of $\sim 3,000 M_{\odot} \mathrm{yr}^{-1}$ if the emission is powered by reprocessed starlight, and the presence of dust and metals may suggest a prior epoch of star formation in the system (Barth et al. 2003; Walter et al. 2003; Bertoldi et al. 2003; Carilli et al. 2004). The general uncertainty about the presence of a massive stellar component in $z \sim 6$ quasars then poses two questions: if the progenitors and descendants of $z \sim 6$ quasars do contain a substantial stellar component what would their properties be and how might one detect them?

The detection of high-redshift galaxy populations at redshifts $z \gtrsim 3$ has greatly developed over the last fifteen years. The adoption of photometric techniques to identify the Lyman break in galaxy spectra (e.g., Steidel \& Hamilton 1992, 1993; Steidel et al. 1996), owing to intergalactic medium (IGM) absorption (e.g.,

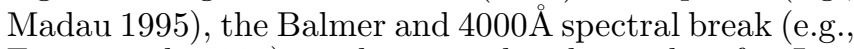
Franx et al. 2003), and narrow-band searches for Ly $\alpha$ emission (e.g. Cowie \& Hu 1998; Hu et al. 2002) has led to the discovery of galaxy populations at progressively higher redshifts. Multicolor Hubble Space Telescope (HST) observations of the Hubble Deep Field (HDF; Williams et al. 1996) allowed for the efficient identification of large numbers of $B_{435}$-band (F435W filter) dropout candidates at $3.5 \lesssim z \lesssim 4.5$ (Madau et al. 1996) and redder, more distant dropout candidates (e.g., Lanzetta et al. 1996) including a $V_{606}$-band $(\mathrm{F} 606 \mathrm{~W}$ filter) dropout spectroscopically verified to reside at $z=$ 5.34 (Spinrad et al. 1998).

Subsequently, photometric dropout techniques based on the Lyman spectral break were used to identify highredshift galaxy populations in the Hubble Ultra Deep Field (HUDF; Beckwith et al. 2006) with the Advanced Camera for Surveys (ACS; Ford et al. 1998) and the Near Infrared Camera and Multi-Object Spectrometer (NICMOS) Ultra Deep Field (Thompson et al. 2005), and in the wider Great Observatories Origins Deep Survey (GOODS; Dickinson et al. 2003; Giavalisco et al. 2004a). As with the HDF data, color selections based on $B_{435^{-}}$and $V_{606}$-dropouts were used (e.g., Giavalisco et al. 2004b), but large samples based on $i_{775}$-band (F775W filter) and even $z_{850}$-band (F850LP filter) dropouts have been identified in GOODS and the HUDF by several groups (e.g., Stanway et al. 2003, 2004b a, 2005; Yan et al. 2003, 2004; Yan \& Windhorst 2004; Yan et al.|2006; Bunker et al.|2003, 2004; Bouwens et al. 2003, 2004; Bouwens \& Illingworth 2006; Bremer et al. 2004; Dickinson et al. 2004; Stiavelli et al. 2004; Evles et al. 2006; Dunlop et al. 2006; Overzier et al. 2006; Lee et al. 2006; Reddy et al. 2006; Vanzella et al. 2006; Verma et al. 2007). The GOODS optical and near-infrared data sets have been combined with Spitzer Space Telescope (Werner et al. 2004) observations using the Infrared Array Camera (IRAC; Fazio et al. 2004) to help constrain longer wavelength portions of the spectral energy distributions (SEDs) of high-redshift galaxy candidates (e.g., Mobasher et al. 2005; Egami et al. 2005; Yan et al. 2005; Eyles et al. 2005; Stark et al. 2006; Papovich et al. 2006; Labbé et al. 2005, 2006, see also Barmby et al. (2004)).

Parallel efforts have been made with ground-based observations, sometimes in concert with HST or Spitzer data sets, including optical break searches (Franx et al. 2003; Daddi et al. 2003; van Dokkum et al. 2004), $z \gtrsim$ 5 Lyman-break galaxy (LBG) searches using a variety of techniques including $R I z$ Lehnert \& Bremer 2003), BVRiz (Ouchi et al. 2004), $B z K_{s}$ (Grazian et al. 2006), and $z S Z J H K$ (Richard et al. 2006) color selections, and very-high redshift $(z \gtrsim 5)$ narrow band and spectroscopic Ly $\alpha$-emitter searches (e.g., Ellis et al. 
2001; Kodaira et al. 2003; Kurk et al.|2004; Santos 2004: Ouchi et al. 2005; Charv et al. 2005; Taniguchi et al. 2005; Stern et al. 2005b; Ive et al. 2006; Kashikawa et al. 2006). Collectively, these observational efforts and others have established the existence of a substantial galaxy population at $z>5$. A brief review of the known highredshift galaxy population can be found in $\mathrm{Hu} \&$ Cowie (2006).

Theoretical calculations of the photometric properties of galaxies have been explored previously by hydrodynamical simulations of cosmological structure formation at redshifts $z<3$ (Katz et al. 1992; Davé et al. 1999; Nagamine et al. 2005a.b), $z \sim 3$ (Weinberg et al. 2002; Nagamine et al. 2004, 2006), $z \geq 4$ (Harford \& Gnedin 2003; Finlator et al. 2006; Night et al. 2006), and $z \gtrsim 6$ (Barton et al. 2004; Davé et al. 2006), and in semi-analytic calculations (e.g., Baugh et al. 1998; Kauffmann et al. 1999; Somerville et al. 2001). These calculations show that simulated galaxies, typically with maximum star formation rates of $\mathrm{SFR} \lesssim 100 M_{\odot} \mathrm{yr}^{-1}$, can satisfy Lyman-break color selection techniques. These simulated galaxy samples broadly agree with the observed properties of high-redshift galaxies, but given the simulated comoving volumes $\left(\lesssim 0.001 h^{-3} \mathrm{Gpc}^{3}\right)$, the galaxies examined to date are orders of magnitudes less massive than $z \sim 6$ quasar host halos that have comoving number densities of $n \sim 1 \mathrm{Gpc}^{-3}$ (Fan et al. 2003). Furthermore, these cosmological simulations have not included prescriptions for energetic feedback from SMBH growth that have been demonstrated to influence the photometric properties of elliptical remnants formed in hydrodynamical simulations of isolated disk galaxy mergers (e.g., Springel et al. 2005a).

To calculate the photometric evolution of $z \sim 6$ quasar host galaxies, we use the simulations of Li et al. (2006) that combine merger trees measured from cosmological N-body simulations of a $1 h^{-3} \mathrm{Gpc}^{3}$ comoving volume with hydrodynamical simulations of galaxy mergers that include gas cooling, star formation and supernovae, and a prescription for feedback from growing SMBHs to track the hierarchical formation of a massive galaxy at redshifts $z \lesssim 14$. These simulations are described in detail in Li et al. (2006) and summarized in 92 . The galaxy hosts a quasar at $z \sim 6.5$ that is similar in bolometric luminosity to the $z=6.42$ quasar SDSS J1148+5251 (Fan et al. 2003). The photometric evolution of the host galaxy is modeled before, during, and after the quasar phase by applying population synthesis models, template AGN spectra, a prescription for dust attenuation and reddening, intergalactic medium absorption, and telescope responses, and is described in 3. In the photometric evolution of the host galaxy is compared with a variety of optical and near-infrared photometric selection criteria from the literature. The observability of high-redshift quasar host galaxies is assessed in 55. We provide a brief discussion in 96 and summarize and conclude in 87 We adopt a flat $\Lambda$ CDM cosmology with matter density $\Omega_{\mathrm{M}}=0.3$, dark energy density $\Omega_{\Lambda}=0.7$, baryon density $\Omega_{\mathrm{b}}=0.04$, spectral index $n=1$, and root-mean-squared fluctuations on $8 h^{-1} \mathrm{Mpc}$ scales of $\sigma_{8}=0.9$. Unless otherwise noted, we report magnitudes on the AB scale Oke 1974).

\section{SIMULATIONS}

We use the Li et al. (2006) hydrodynamical simulation of the formation of a $z \sim 6$ quasar as input for our calculation of the photometric evolution of the most massive quasar host galaxies. The simulation is described in detail by Li et al. (2006), and other specifics of the modeling can be found in Springel et al. (2005b) and Robertson et al. (2006b), but a brief summary of the simulation follows.

The rarity of $z \sim 6$ quasars requires the simulation of very large volumes to capture the high-sigma fluctuations that give rise to early-forming massive galaxies. To accomplish this, a $400^{3}$ particle N-body simulation of a $1 h^{-3} \mathrm{Gpc}^{3}$ volume of a cosmology consistent with the first-year results of the Wilkinson Microwave Anisotropy Probe (WMAP1, $\Omega_{\mathrm{M}}=0.3, \Omega_{\Lambda}=0.7, \Omega_{\mathrm{b}}=0.04, \sigma_{8}=$ 0.9, Spergel et al. 2003) was performed. This simulation, with dark matter particle mass $m_{\mathrm{DM}}=1.3 \times 10^{12} h^{-1} M_{\odot}$ and force resolution $\epsilon=125 h^{-1} \mathrm{kpc}$, was evolved from $z \sim 30$ to $z \sim 0$. Dark matter particles bound to the most massive halo in the volume, with $M_{\text {halo }}=$ $3.6 \times 10^{15} h^{-1} M_{\odot}$ at $z=0$, are identified at $z \approx 6$. The $\mathrm{Gpc}^{3}$ volume is then resimulated (Power et al. 2003; Gao et al. 2005) from $z=69$ to $z=0$ with $350^{3}$ dark matter particles, with $340^{3}$ high-resolution dark matter particles $\left(M_{d m}=2.8 \times 10^{8} h^{-1} M_{\odot}, \epsilon=5 h^{-1} \mathrm{kpc}\right)$ distributed in the $\sim 1.25 \times 10^{-4} h^{-3} \mathrm{Gpc}^{3}$ Lagrangian volume that comprised the most massive halo at $z \sim 6$ and the remaining particles properly placed throughout the rest of the $\mathrm{Gpc}^{3}$ volume to retain the large scale tidal field.

The $\Lambda$ CDM simulation is used to follow each merger in the hierarchical formation history of the halo between $z=14.4$ and $z=6$. The merger history is comprised of 7 major mergers (with mass ratios less than $5: 1$ ) between $z=14.4$ and $z=8.5$ contributing to the $5.4 \times 10^{12} h^{-1} M_{\odot}$ halo mass at $z \sim 6$. To model the formation of the baryonic component of the quasar host halo, each event in the merger tree is resimulated using the smoothed particle hydrodynamics (SPH) / N-body code GADGET2 (Springel 2005). The merging systems are represented with hydrodynamical models of disk galaxies (Springel et al. 2005b) with structural properties initialized according to the Mo et al. (1998) dissipational galaxy formation formalism and scaled appropriately to high-redshift following Robertson et al. (2006b). The scalings simply fold the redshift- and mass-dependent dark matter concentrations (e.g. Bullock et al. 2001) and the redshift-dependent Hubble parameter $H(z)$ through the Mo et al. (1998) formalism, resulting in smaller, more centrally concentrated disks at higher redshifts. The orbital parameters of each progenitor galaxy (see Table 1 of $\mathrm{Li}$ et al. (2006)) are set such that the initial separation of the galaxies is the current host halo virial radius at the time of the merger and the pericentric passage distance is half the progenitor disk scale-length $R_{\text {peri }}=0.5 R_{\mathrm{d}}$.

The merging disk galaxies range in virial mass from the initial progenitor's $M_{\text {vir }}=6.3 \times 10^{10} h^{-1} M_{\odot}$ at $z=14.4$ to the last merging galaxy's $M_{\text {vir }}=2.08 \times 10^{12} h^{-1} M_{\odot}$. Each progenitor disk has a mass $m_{\mathrm{d}}=0.15 M_{\text {vir }}$, with gas fractions of $f_{\text {gas }}=1$ at redshifts $z \geq 10$ and $f_{\text {gas }}=0.9$ at redshifts $8 \leq z<10$. For the numerical parame- 
ters of the progenitor galaxy models, a fixed dark matter particle mass $\left(M_{\mathrm{dm}}=1.1 \times 10^{7} h^{-1} M_{\odot}\right)$ and baryonic particle mass $\left(M_{\text {gas }}=M_{\text {star }}=2.2 \times 10^{6} h^{-1} M_{\odot}\right)$ is used. The gravitational softening for the dark matter particles $\left(\epsilon_{\mathrm{dm}}=60 h^{-1} \mathrm{pc}\right)$ is set twice as large as for the baryonic particles $\left(\epsilon_{\text {gas.stars }}=30 h^{-1} \mathrm{pc}\right)$. Star formation is treated in a McKee \& Ostriker (1977) multiphase picture for the interstellar medium (ISM), implemented as a numerical algorithm by Springel \& Hernquist (2003). In this multiphase ISM model, dense, star-forming gas particles are treated as a collection of cold molecular clouds embedded in a tenuous phase heated by supernovae. The starformation rate is calibrated to reproduce the SchmidtKennicutt Law (Schmidt 1959; Kennicutt 1998), with the star forming gas mass calculated from the cold phase density. Stellar particles are spawned probabilistically from star- forming gas particles. Each stellar population is formed with half the mass of its parent gas particle and recycles $2 \%$ of its mass as metals through supernovae (for details, see Springel \& Hernquist 2003).

The Li et al. (2006) simulation includes a prescription for SMBH accretion and feedback (for details, see Springel et al. 2005b). Each simulated galaxy is initialized with a SMBH "sink" particle that is allowed to spherically accrete through a Bondi (1952) prescription. The accretion rate is determined from the gas temperature and density near the SMBH particle, and is limited to the Eddington rate. $\mathrm{A}$ fraction $\epsilon_{r}=0.1$ of the accreted rest mass energy released as radiation and a small fraction of this radiated energy (5\%) is coupled to the surrounding gas as thermal feedback. The strength of the feedback is calibrated to reproduce the local $M_{\mathrm{BH}}-\sigma$ relation in isolated mergers of disk galaxies (Di Matteo et al. 2005). The mass of the SMBH seed in each galaxy is determined by assuming a single $200 M_{\odot}$ black hole formed in each halo at $z=30$, in accord with expectations from Population III star formation (e.g. Abel et al. 2002; Bromm \& Larson 2004; Yoshida et al. 2003, 2006), and then experienced Eddington-limited growth until its host galaxy merged into the quasar host halo. The $\mathrm{SMBH}$ seeds initialized in this manner range in mass from $M_{\mathrm{BH}}=1.5 \times 10^{4} h^{-1} M_{\odot}$ at $z=14.4$ to $M_{\mathrm{BH}}=8.92 \times 10^{6} h^{-1} M_{\odot}$ at $z=8.5$. As dynamical friction operates on the merging galaxies during the simulation, the central regions of each system eventually coalesce. Once the SMBH particles approach to within the gravitational force resolution they are merged.

\subsection{Mergers, Star Formation Rate, and Active Galactic Nuclei Luminosity}

The seven major mergers between eight galaxies occur during five redshift eras of the evolution: the first merger between two galaxies $\left(M_{\mathrm{vir}, 1}=5.3 \times 10^{10} h^{-1} M_{\odot}\right.$, $\left.M_{\mathrm{vir}, 2}=6.3 \times 10^{10} h^{-1} M_{\odot}\right)$ begins at $z=14.4$, followed by two additional mergers $\left(M_{\mathrm{vir}, 3}=1.5 \times 10^{11} h^{-1} M_{\odot}\right.$, $\left.M_{\text {vir }, 4}=1.77 \times 10^{11} h^{-1} M_{\odot}\right)$ at $13.0>z>10.5$, single mergers at $10.5>z>9.4\left(M_{\mathrm{vir}, 5}=4.91 \times 10^{11} h^{-1} M_{\odot}\right)$ and at $9.4>z>8.5\left(M_{\mathrm{vir}, 6}=7.96 \times 10^{11} h^{-1} M_{\odot}\right)$, and two final mergers $\left(M_{\mathrm{vir}, 7}=1.6 \times 10^{12} h^{-1} M_{\odot}\right.$, $\left.M_{\text {vir }, 8}=2.08 \times 10^{12} h^{-1} M_{\odot}\right)$ at $z \lesssim 8.5$. The fresh gas supply introduced by each merger, combined with the high density environment and violent tidal interactions,

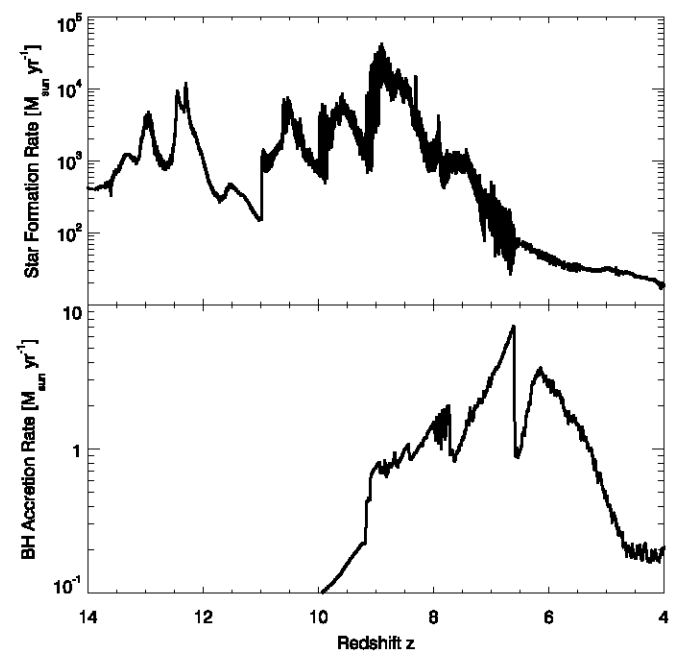

FIG. 1.- Star formation rate (SFR) and supermassive black hole $(\mathrm{SMBH})$ accretion rate as a function of redshift in the Li et al. (2006) simulation of the hierarchical formation of a $z \sim 6$ quasar. The upper panel shows the SFR determined from the density of cold gas over the course of eight galaxy mergers in the build-up to quasar activity at $z \sim 6.5$. The lower panel shows the accretion rate $\dot{M}_{\mathrm{BH}}$ onto SMBHs in the system, determined through a Bondi (1952) spherical accretion model that depends on the properties of gas near the SMBH particles (for details, see Springel et al. 2005b). The unattenuated bolometric luminosity of the active galactic nuclei (AGN) fueled by SMBH accretion can be calculated from the accretion rate $\dot{M}_{\mathrm{BH}}$. Adapted from Li et al. (2006).

leads to peaks in the star formation rate (SFR, Fig. 1 upper panel) that temporally correlate with the merger events. The SFR peaks near the $z \sim 8.5$ mergers with $\mathrm{SFR}>10^{4} M_{\odot \mathrm{yr}^{-1}}$, but frequently exceeds $10^{3} M_{\odot \mathrm{yr}^{-1}}$ over the redshift range $8<z<13$.

The total SMBH accretion rate $\dot{M}_{\mathrm{BH}}$ (Fig. 1, lower panel) also influences the observable properties of the system. The unattenuated bolometric luminosity $L_{\mathrm{bol}}$ of the active galactic nuclei (AGN) powered by the growing $\mathrm{SMBHs}$ is determined by the accretion rate onto the SMBHs through the equation

$$
L_{\mathrm{bol}}=\epsilon_{r} \dot{M}_{\mathrm{BH}} c^{2}=1.45 \times 10^{12}\left(\frac{\epsilon_{r}}{0.1}\right)\left(\frac{\dot{M}_{\mathrm{BH}}}{M_{\odot} \mathrm{yr}^{-1}}\right) L_{\odot}
$$

where $\epsilon_{r}=0.1$ is the radiative efficiency of the accretion model we adopt and $c$ is the speed of light.

\section{PHOTOMETRIC MODELING}

Modeling the observed spectral energy distribution (SED) of the quasar host galaxy involves calculating the SEDs of each individual stellar population and AGN present in the system and applying a wavelengthdependent dust correction to the calculated SEDs that accounts for internal interstellar absorption. The emission from the galaxy, attenuated by ISM reddening, must then be corrected for both the IGM opacity owing to Lyman series absorption and redshifting of the SED owing to cosmological expansion. The measured photometry of the object is then modified by the combined wavelength- 
dependent transmission of the filter and telescope assembly as well as the wavelength-dependent quantum efficiency of the detector. Below we describe how we model each contribution to the observable photometric properties of the massive quasar host galaxy.

\subsection{Stellar Population Synthesis}

The hydrodynamical simulation follows the formation of individual stellar populations formed from dense gas, as described in \$2. Each stellar particle represents several million solar masses of stars, so an initial mass function (IMF) must be selected to represent distribution of stellar masses formed when each stellar particle is spawned. The IMF also influences the spectral evolution of each stellar population through its time-dependent mass-to-light ratio. We adopt the Kroupa (2001, 2002) IMF, which describes the number $N$ of stars of a given mass $m$ as a power-law $\mathrm{d} N / \mathrm{d} m \propto m^{-s}$. The Kroupa IMF shares the same power-law index $s \approx 2.3$ as the Salpeter (1955) IMF for $m \geq 0.5 M_{\odot}$ but flattens to $s=1.3$ at lower masses. The mass-to-light ratio of the Kroupa IMF is correspondingly lower than for the Salpeter (1955) IMF, by a factor of $\sim 2$, when normalized over the mass range $0.1 M_{\odot} \leq m \leq 100 M_{\odot}$

The metallicity and age of each stellar particle is tracked in the simulation, allowing for the use of stellar population synthesis models to calculate an evolving SED for each particle. We use Starburst99 (Leitherer et al. 1999; Vázquez \& Leitherer 2005) to model the spectra from each population, given the mass, age, metallicity, and assumed Kroupa IMF. The version of Starburst99 we use includes the Padova stellar evolution tracks (e.g., Fagotto et al. 1994) to improve the performance of Starburst99 for older $(t>0.1 \mathrm{Gyr})$ stellar populations (for details, see Vázquez \& Leitherer 2005). Illustrations of the time-dependent SEDs produced by Starburst99 can be found in Figures 4 and 5 of Vázquez \& Leitherer (2005). In practice, we produce a grid of Starburst99 spectral models at wavelengths $0.0091 \mu \mathrm{m} \leq \lambda \leq 160 \mu \mathrm{m}$ for stellar populations over the range of ages $t=10^{4}-10^{10} \mathrm{yr}$, spaced logarithmically, and for metallicities of $Z=0.02-2.5 Z_{\odot}$, and then interpolate the Starburst99 models to produce an SED appropriate for each stellar particle.

\subsection{Active Galactic Nuclei SEDs}

As discussed in $\$ 2$, the simulations follow accretion onto SMBH particles. Given the radiative efficiency we adopt $\left(\epsilon_{r}=0.1\right)$, we can calculate the bolometric luminosity of the AGN associated with each SMBH (Eq. 1). According to the feedback prescription by Springel et al. (2005b), a fraction $\eta=0.05$ of this bolometric luminosity is injected as thermal energy in the surrounding gas. The remaining $\epsilon_{r}(1-\eta)$ fraction of the radiated energy would be visible as nuclear emission in the absence of intervening material. To model this emission, we combine the power-law + x-ray reflection component SED template for AGN from Marconi et al. (2004) with the average quasar emission template from Vanden Berk et al. (2001).

The Marconi et al. (2004) SED template consists of a power-law $L_{\nu} \propto \nu^{\alpha}$, with $\alpha=-0.44$ in the range $0.13 \mu \mathrm{m}<\lambda<1 \mu \mathrm{m}$ set to match the slope inferred from stacked SDSS quasar spectra (Vanden Berk et al. 2001), and a Rayleigh-Jeans fall-off $(\alpha=2)$ at longer wavelengths. The ultraviolet $(0.05 \mu \mathrm{m}<\lambda<0.12 \mu \mathrm{m})$ emission has a power-law slope $(s=-1.76)$ determined by Telfer et al. (2002) based on the HST spectra of $\sim 200$ quasars, while the x-ray emission at energies above $1 \mathrm{keV}$ is modeled with a power-law plus PEXRAV model (Magdziarz \& Zdziarski 1995) reflection component. The normalization of the x-ray component relative to the optical power-law component is fixed to reproduce the quantity $\alpha_{\mathrm{OX}}$ (Zamorani et al. 1981) that relates the ultraviolet $(\lambda \approx 0.25 \mu \mathrm{m})$ to $\mathrm{x}$-ray $(E \approx 2 \mathrm{keV})$ flux ratio. The Marconi et al. (2004) SED template uses a luminosity-dependent $\alpha_{\mathrm{OX}}$ following Vignali et al. (2003) that leads to a decreasing fraction of the bolometric luminosity emitted in x-rays as the AGN luminosity increases. The ratio of bolometric luminosity to $B$-band luminosity $L_{\mathrm{bol}} / \nu_{B} L_{\nu, B}$ has a corresponding decrease with bolometric luminosity. Figure 3 of Marconi et al. (2004) presents illustrations of their AGN SED template and luminositydependent $B$-band and x-ray bolometric corrections.

Vanden Berk et al. (2001) produced a composite quasar spectrum by stacking the spectra of more than 2000 SDSS quasars at wavelengths $0.08 \mu \mathrm{m}<\lambda<$ $8555 \mu \mathrm{m}$, including systems with $r^{\prime}$ absolute magnitudes of $-18.0>r^{\prime}>-26.5$ over the redshift span $0.044 \leq z \leq$ 4.789. The continuum of the geometric mean spectrum of these quasars can be represented by a broken powerlaw $L_{\nu} \propto \nu^{s}$ with $s=-0.44$ over the wavelength range $0.13 \mu \mathrm{m}<\lambda<0.5 \mu \mathrm{m}$ and $s=-2.45$ at longer wavelengths, and is representative of the composite spectrum of $z \sim 6$ quasars (Fan et al. 2004). While the short wavelength slope is flatter than the slope $s \approx-0.9$ found for high-redshift quasars (Schneider et al. 1991; Fan et al. 2001b; Schneider et al. 2001), the difference can be accounted for by difficulties in determining the continuum. Prominent emission lines in the Vanden Berk et al. (2001) template spectrum include Ly $\alpha$, CIV, MgII, and $\mathrm{H} \alpha$.

To combine the Marconi et al. (2004) SED template with the Vanden Berk et al. (2001) quasar spectrum, we replace the optical power-law component of the Marconi et al. (2004) with the Vanden Berk et al. (2001) spectrum over wavelengths $0.11 \mu \mathrm{m}<\lambda<0.86 \mu \mathrm{m}$ and match the Rayleigh-Jeans and UV power-law continua to the emission line spectrum. The spectrum is then integrated and renormalized to maintain the desired bolometric luminosity, and approximately maintains the emission line equivalent widths and Marconi et al. (2004) luminosity-dependent bolometric corrections while scaling the emission line flux with the AGN luminosity. As discussed below, the choice to combine these spectral templates only influences our results during the evolutionary phases when the AGN dominates the emission of the system, over small redshift ranges near $z \approx 6.5$ and $z \approx 6$ (see Fig. 1 above and Fig. 11 of Li et al. 2006), but allows us to incorporate the effect of $\mathrm{Ly} \alpha$ emission on quasar color selection (e.g., Fan et al. 2001a).

\subsection{Interstellar Reddening and Absorption}

The methods for modeling the emission from stellar populations (\$3.1) and AGN (\$3.2) allow for an estimation of the unattenuated SEDs of simulated galaxies. The gas-rich galaxies involved in the mergers that lead 
to quasar activity at $z \sim 6$ contain substantial columns of hydrogen and, owing to the efficient star formation in dense gas, large amounts of obscuring dust. The wavelength-dependent attenuation from dust reddening should therefore be accounted for when modeling the observed SED of the quasar host galaxy.

To incorporate dust attenuation in the photometric modeling, the hydrogen column density $N_{H}$ and an estimate of the gas metallicity is calculated along the lineof-sight to each stellar population. We use the method of Hopkins et al. (2005) to calculate the gas density on a fine grid from the SPH formalism and the particle distribution. For dense gas above the threshold for star formation a two-phase decomposition of the Springel \& Hernquist (2003) multiphase ISM model is performed, assuming the hot and cold phases are in pressure equilibrium, in order to calculate the hot phase column density. For each AGN in the system the column density and metallicity is measured along 100 lines-ofsight to calculate a distribution of optical depths, allowing for an estimate of the scatter in the internal galactic absorption. For discussions on general agreement between the bound-free and metal-line absorption calculated from this method for estimating $N_{H}$ columns to AGN and models of the cosmic x-ray background, see, e.g., Hopkins et al. (2006a, 2007b).

Once the $N_{H}$ columns and gas metallicities are calculated, the visual attenuation $A_{V}$ owing to dust is calculated by scaling the Milky Way (MW) dust-to-gas ratio $A_{V} / N_{H}=5.35 \times 10^{-22}$ (Bohlin et al. 1978; Cox 2000) by the gas metallicity. A linear scaling with metallicity, normalized to the MW ratio for solar metallicity gas, roughly accounts for the observed dust-to-gas ratios in the less metal-rich Large Magellanic Cloud (LMC) and Small Magellanic Cloud (SMC) (e.g., Fitzpatrick 1986; Rodrigues et al. 1997). The wavelength-dependent dust attenuation $A_{\lambda} / A_{V}$ must also be modeled, with possible choices including the MW dust curve (e.g., Cardelli et al. 1989) that includes the strong $0.22 \mu \mathrm{m}$ absorption feature, LMC and SMC dust (e.g., Pei 1992; Gordon et al. 2003), and the possibly supernovae-related dust in highredshift quasars (Maiolino et al. 2004). We adopt the dust model advocated by Calzetti et al. (1994) for modeling the UV emission from starburst galaxies, with the updated $R_{V}=4.05$ from Calzetti et al. (2000) (see also Calzetti 1997). The Calzetti et al. (2000) dust law is frequently used to model LBGs and infer their stellar masses (e.g., Papovich et al. 2001; Shapley et al. 2005), and estimates of unattenuated star formation rates using Calzetti et al. (2000) dust corrections have been used to successfully predict the x-ray and radio properties of galaxies at $z \sim 2$ (Reddy \& Steidel 2004). Using SMC dust would increase the UV absorption of the model spectra and increase the transition redshift where model spectra cross boundaries in color-color selections designed to be sensitive to the Lyman break, but would not change the photometric selection of the model spectra with a given color criterion over most of the system's redshift evolution.

\subsection{Intergalactic Medium Absorption}

Intervening hydrogen clouds in the intergalactic medium (IGM) can greatly attenuate flux via Lymanseries absorption. To account for this IGM opacity, we adopt the Madau (1995) model of Poisson-distributed absorbers in which Lyman series blanketing causes a "staircase" absorption profile blue-ward of Ly $\alpha$. The spectral break introduced by this absorption has been frequently used to engineer the photometric selection of highredshift galaxies (e.g., Madau et al. 1996; Steidel et al. 1996; Giavalisco et al. 2004b). By $z \approx 4.5$, the mean transmission is only $30 \%$ near Ly $\alpha$, but the Poissonian nature of the model can lead to substantial variation in the optical depth $\tau$ at intermediate redshifts. At $z \sim 3.5$ the $\pm 1 \sigma$ fluctuation in optical depth corresponds to $30 \%$ transmission variation relative to the unattenuated spectra (see, e.g., Fig. 3 of Madau 1995). However, Monte Carlo simulations suggest that the bandpass-averaged fluctuations in transmission are typically smaller $(\sim 0.1$ magnitudes, see, e.g., Bershady et al. 1999). At high redshifts $(z \gtrsim 6)$ the absorption is very strong near Ly $\alpha$ (e.g., $\tau>3$, see Fig. 2 of Night et al. 2006) and typical line-of-sight fluctuations will lead to strong spectral breaks. We therefore assume the mean IGM opacity calculated from the model since we are primarily concerned with high-redshifts $(z \gtrsim 5)$, but we note that the calculated photometric trajectories could experience either small shifts in color-color space position or rate of change with redshift owing to line-of-sight opacity fluctuations.

\subsection{Telescope, Filter, and Detector Response}

To calculate the observed photometric properties of simulated galaxies, the wavelength-dependent transmission of telescope assemblies, filters, and detector quantum efficiency (QE) must be modeled together to determine the net transmissivity of a given observational facility.

For the SDSS $u^{\prime}-, \quad g^{\prime}-, \quad r^{\prime}-, \quad i^{\prime}-$, and $z^{\prime}$-band (Fukugita et al. 1996) transmissivities, we use the USNO40 response functions measured by Jim Gunn ${ }^{6}$. The calculated $U_{n}, G$, and $R_{s}$ (Steidel \& Hamilton 1993) magnitudes use the transmissivity measured with the KPNO Mosaic CCDs ${ }^{7}$. For comparison with HST observations, we calculated the wavelength response curves from the combined transmissivity of the mirror and filters with the detector QE for F435W, F606W, F775W, F814W, and F850LP observations with ACS ${ }^{8}$ and F110W and F160W observations with NICMOS ${ }^{9}$. For the ground-based $J_{-}, H$-, and $K_{s}$-band transmissivities, we use the calculated relative response curves for $2 \mathrm{MASS}$ from Cohen et al. (2003). The IRAC throughput response was adopted from data provided by the Spitzer Science Center ${ }^{10}$. In practice, over the redshift range $(z \gtrsim 4)$ considered at flux in all filters bluer than F435W were always too heavily absorbed to be easily measured (>30 AB magnitudes), so in what follows we focus on the observable properties of galaxies in redder filters.

\subsection{Combined SED Modeling}

The final model SEDs calculated for the simulated quasar host galaxy combines the stellar population synthesis models described in $\$ 3.1$, the AGN spectral tem-

\footnotetext{
${ }^{6}$ http://www-star.fnal.gov/ugriz/Filters/response.html

7 http://www.noao.edu/kpno/mosaic/filters/filters.html

$8 \mathrm{ftp}: / / \mathrm{ftp}$. stsci.edu/cdbs/cdbs2/comp/acs/

9 ftp://ftp.stsci.edu/cdbs/cdbs2/comp/nicmos/

$10 \mathrm{http://ssc.spitzer.caltech.edu/irac/spectral \_ response.html}$
} 


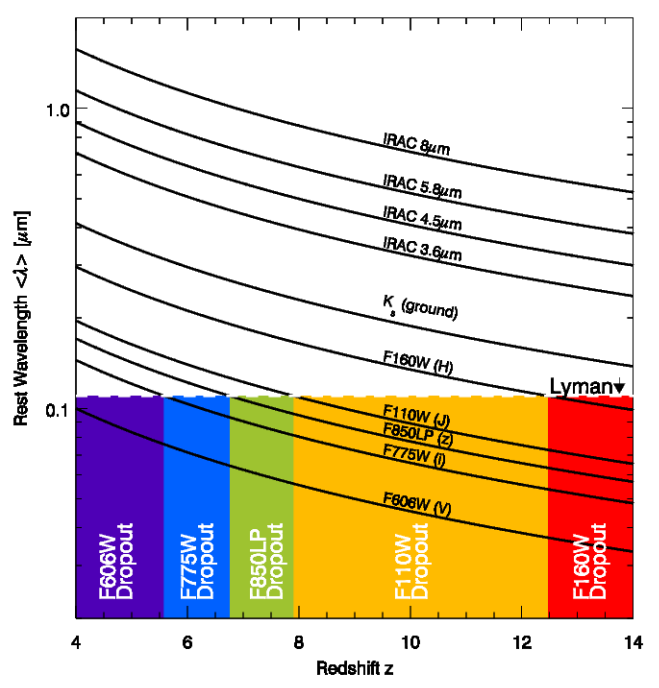

FIg. 2.- Schematic diagram of Ly $\alpha$-break dropouts in optical and near-infrared filters (F606W, F775W, F850LP, F110W, F160W, $K_{s}, 3.6 \mu \mathrm{m}, 4.5 \mu \mathrm{m}, 5.8 \mu \mathrm{m}$, and $\left.8.0 \mu \mathrm{m}\right)$ at redshifts $z \sim 4-14$. Cosmological expansion enables intergalactic medium (IGM) absorption owing to hydrogen Lyman-series opacity (e.g., Madau 1995) to absorb light at progressively longer observed wavelengths. As the Lyman-

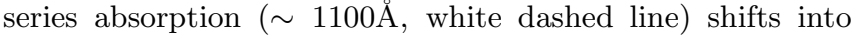
redder filters, galaxies begin to drop out of the corresponding photometric samples (shaded regions represent a $>20 \%$ flux decrement in a given band for a flat spectrum). The solid lines show the effect of cosmological redshifting on the rest-frame wavelength coverage of commonly used filters.

plate described in 3.2 ISM attenuation described in 3.3. and IGM attenuation described in 3.4 to arrive at the observed flux density (adapting the notation of Papovich et al. 2001)

$$
\begin{aligned}
F_{\nu}(z, \lambda)= & (1+z) \frac{\sum_{i}^{N} L_{\nu_{0}, i}\left(\lambda_{0}, t_{i}, A_{\lambda_{0}, i}, M_{\star, i}, \dot{M}_{\mathrm{BH}, i}\right)}{4 \pi D_{L}^{2}(z)} \\
& \times e^{-\tau_{\mathrm{IGM}}(z, \lambda)},
\end{aligned}
$$

where the rest-frame emission $L_{\nu_{0}, i}$ as a function of restwavelength $\lambda_{0}$ from each of $N$ sources may depend on the its stellar age $t_{i}$, stellar mass $M_{\star, i}$, or SMBH accretion rate $\dot{M}_{\mathrm{BH}, i}$, and the dust attenuation $A_{\lambda_{0}, i}$ along the line-of-sight to each source. The typical number of individual stellar population sources is $N \gtrsim 750,000$ by $z \sim 6$. The total emitted spectrum from the galaxy is attenuated by the IGM optical depth $\tau_{I G M}$, and the factors of $(1+z)$ and the luminosity distance $D_{L}(z)$ account for the cosmology-dependent redshift and distance. Again following Papovich et al. (2001), the flux density in each filter is calculated as

$$
\left\langle F_{\nu}(z)\right\rangle=\frac{\int T_{\nu} F_{\nu}(z, \lambda) \mathrm{d} \nu / \nu}{\int T_{\nu} \mathrm{d} \nu / \nu}
$$

where unit-free $T_{\nu}$ represents the combined transmissivity of the telescope and filter and the detector QE, as described in 3.5. The flux density is converted into a magnitude on the AB scale (Oke 1974) through the relation

$$
m_{\mathrm{AB}}=-2.5 \log \left(\frac{\left\langle F_{\nu}\right\rangle}{1 \mu \mathrm{Jy}}\right)+23.9
$$

where $\left\langle F_{\nu}(z)\right\rangle$ is the bandpass-weighted flux density (Eq. (3) and $1 \mu \mathrm{Jy}=10^{-29} \mathrm{ergs} \mathrm{cm}^{-2} \mathrm{~s}^{-1} \mathrm{~Hz}^{-1}$.

The machinery described above allows for a thorough determination of the photometric properties of a simulated galaxy and an application of common color "dropout" selection techniques based on spectral breaks to track the photometric evolution of the quasar host galaxy with redshift. For reference, a schematic diagram of the relevant redshifts for dropout samples in the ACS and NICMOS filters and a characteristic wavelength coverage for optical and near-IR filters with redshift is provided in Figure 2

\section{PHOTOMETRIC EVOLUTION}

The photometric evolution of the quasar host galaxy is calculated using the methods described in $\$ 3$, In our modeling, we track the luminosity of only the main host halo of the quasar during its evolution; the luminosities of the merging systems are not followed before they enter the host halo virial radius. The merging systems are modeled as almost completely gaseous, resulting in a smooth photometric evolution as each new system is added to the simulation. The variations in the system brightness owe to increases in the star formation rate during the mergers and the evolution of the combined stellar populations of the quasar host galaxy. Below, we describe the photometric evolution of the simulated system over the redshifts $z=4-14$.

Figure 3 shows the SED and 15 magnitudes calculated for the galaxy at six characteristic times $(z \sim 12$, $z \sim 10, z \sim 8.5, z \sim 6.5, z \sim 5$, and $z \sim 4$ ) during three distinct phases in its evolution. At redshifts $z \gtrsim 7$, the system rapidly forms stars as the progenitors of the quasar host galaxy merge into the system. Each of these gas-rich mergers adds enough fuel to induce large star formation rates of SFR $\gtrsim 10^{3} M_{\odot} \mathrm{yr}^{-1}$. This starburst phase coincides with the formation epoch of the stars that later form the quasar host galaxy. Over this timespan the stellar mass in the system has grown to $M_{\star}=1.595 \times 10^{12} M_{\odot}$, or $96 \%$ of the final stellar mass at $z=4$, with a mass-weighted mean stellar age that increases from $\langle t\rangle=124 \mathrm{Myr}$ at $z=9$ to $\langle t\rangle=185 \mathrm{Myr}$ at $z=7.5$. The upper panels of Figure 3 show the characteristic starburst spectrum of the galaxy during this phase, with strong rest-frame UV and blue emission. The SED evolves most strongly at long rest-frame wavelengths as the mean stellar age of the system increases, but the short wavelength rest-frame emission is remarkably similar during this phase. The luminosity of the system increases as the star formation rate and stellar mass both increase to $z \sim 8$. The large optical depth owing to Lyman-series absorption causes a near step-function break blue-ward of the Ly $\alpha$ line.

By $z \sim 7.5$, the quasar host galaxy begins to change rapidly. At this time a total gas mass of $M_{\text {gas }}=$ $1.21 \times 10^{11} M_{\odot}$ remains, which amounts to roughly $7 \%$ of the total baryonic mass of the system. Of this gas, $30 \%$ has already been shocked, heated by feedback, or is otherwise too diffuse to form stars (e.g., Cox et al. 2006a). The remaining $5 \%$ of the star-forming baryons is dense enough to support a large star formation rate of SFR $\sim 1000 M_{\odot} \mathrm{yr}^{-1}$. However, the starburst epoch is clearly over, extinguished primarily by the consumption of the available fuel rather than by some regula- 


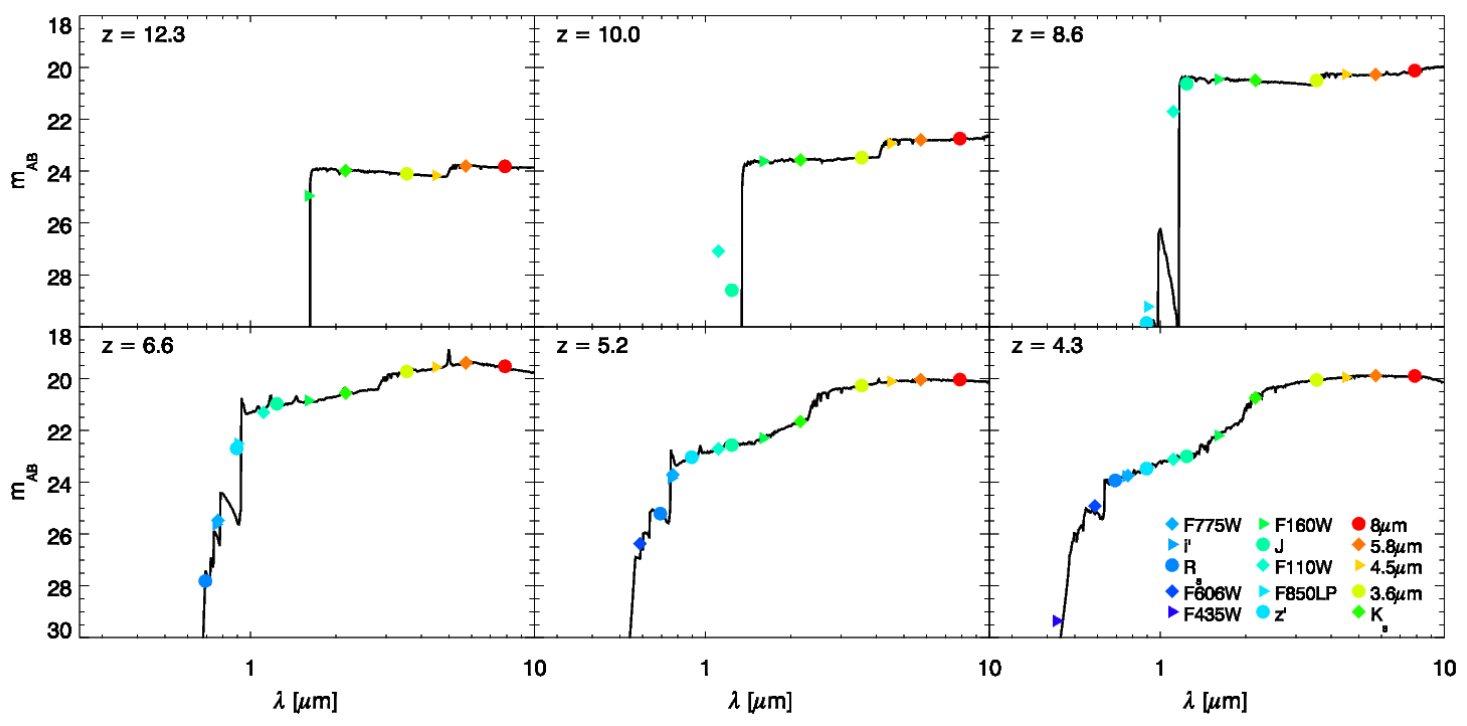

FIG. 3.- Photometric evolution of the quasar host galaxy with redshift. The spectral energy distributions (SEDs), plotted in the observed frame, are calculated from stellar and active galactic nuclei template spectra, attenuated for interstellar absorption, Lyman-series absorption in the intergalactic medium, and cosmological redshifting and distance (see 33 ). At early times $(z \sim$ $8.5-12$, upper panels), the galaxy is rapidly star-forming and has ultraviolet-bright rest-frame spectra typical of starburst galaxies. By $z \sim 6.5$ (bottom left panel), the SMBH accretion has peaked, leading to a luminous quasar phase that satisfies the Sloan Digital Sky Survey quasar color selection. As the SMBH accretion drops and the massive bulge ages $(z \lesssim 6$, bottom middle and right panels), the quasar host galaxy becomes a progressively redder luminous galaxy with a large Balmer/4000 break indicative of an aging stellar population. The large bandwidth of some filters can cause an overestimate of the true flux density when they straddle the Ly $\alpha$ absorption line (e.g., F110W at $z \sim 10$ ).

tory feedback mechanism. As the star formation rate rapidly declines, the stellar population quickly ages and increases to a mean age of $\langle t\rangle=250 \mathrm{Myr}$ by $z \sim 7$ and $\langle t\rangle=407 \mathrm{Myr}$ by $z \sim 6$. While the gas supply cannot sustain the formation of a large new population of stars, it can easily support the exponential growth of SMBHs to $M_{\mathrm{BH}} \sim 10^{9} M_{\odot}$. As the violent merging of the system continues to drive the remaining dense gas toward the galactic centers a rapid phase of Eddington-limited SMBH growth begins and eventually leads to a luminous quasar phase at $z \sim 6.5$ after the SMBHs particles coalesce. The SED of the galaxy reflects the quasar activity, with the hybrid Vanden Berk et al. (2001)-Marconi et al. (2004) model AGN spectrum dominating the blue emission of the galaxy at $z \approx 6.6$ (Fig. 3. lower left panel). The massive stellar component of the system, with a mean stellar age of $\sim 100 \mathrm{~s} \mathrm{Myr,}$ contributes substantially to the emission at rest-frame wavelengths $\lambda_{0}>0.3 \mu \mathrm{m}$ and instills the SED with a Balmer $/ 4000 \AA$ break between the $K_{s^{-}}$and $3.6 \mu \mathrm{m}$-bands. As feedback from the SMBH heats the gas in the centralmost regions of the quasar host galaxy, the fuel source for SMBH accretion is depleted and the prominence of the AGN contribution to the SED declines. By $z \approx 5.2$ (Fig. 3. lower middle panel) the massive old stellar population with mean stellar age $\langle t\rangle=588 \mathrm{Myr}$ dominates the spectrum, with residual SMBH accretion providing lessdominant AGN activity. The rest-frame UV and blue optical portion of the SED becomes increasingly feeble compared with the observed near-IR spectral region powered by mature stars.

Below $z \sim 5$, the host galaxy continues to age and redden during its final, passive evolutionary phase. The star formation rate has declined by approximately three orders of magnitude from its peak near redshift $z \sim 9$. By $z \approx 4.3$ (Fig. 3, lower right panel) the massive stellar component has reached a mean age of $\langle t\rangle=873 \mathrm{Myr}$, and by $z \approx 4$ the mean stellar age will increase to $\langle t\rangle=1.01 \mathrm{Gyr}$. The UV and blue optical emission from the galaxy has correspondingly decreased, leaving a SED

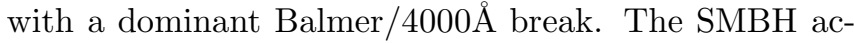
cretion rate has dropped to $\dot{M}_{\mathrm{BH}} \sim 0.2 M_{\odot} \mathrm{yr}^{-1}$, bringing the bolometric luminosity of the AGN activity to $\sim 10 \%$ of the bolometric luminosity of the stellar spheroid. If the system were to continue to evolve passively to low redshift, the galaxy would continue to redden. The giant spheroid would eventually become too red to appear in LBG samples, such as those defined by $U_{n}-G$ (Steidel \& Hamilton 1993; Steidel et al. 1995) and $U_{300}-$ $B_{450}$ (Madau et al. 1996; Steidel et al. 1996) criteria, but would be identified in Distant Red Galaxy (DRG) samples (e.g., Franx et al. 2003) with $\left(J-K_{s}\right)=3.2$ and $\left(I-K_{s}\right)=5.6$ (Vega) at $z \sim 3$. The quasar descendant would eventually obtain a $g^{\prime}-r^{\prime}=1.24$ color (calculated assuming passive evolution to $z \sim 0.2$ ) typical of brightest cluster galaxies in the MaxBCG cluster catalogs of the SDSS (e.g. Koester et al. 2007).

The SEDs calculated for each of the three phases in the photometric evolution of the quasar host galaxy can be used to follow the trajectory of the system through various color-color spaces. The magnitudes calculated according the method described in 3.5 can be compared with color criteria from the literature to determine whether color-selected samples would find massive quasar host galaxies. In what follows, we examine whether the simulated galaxy satisfies common color 


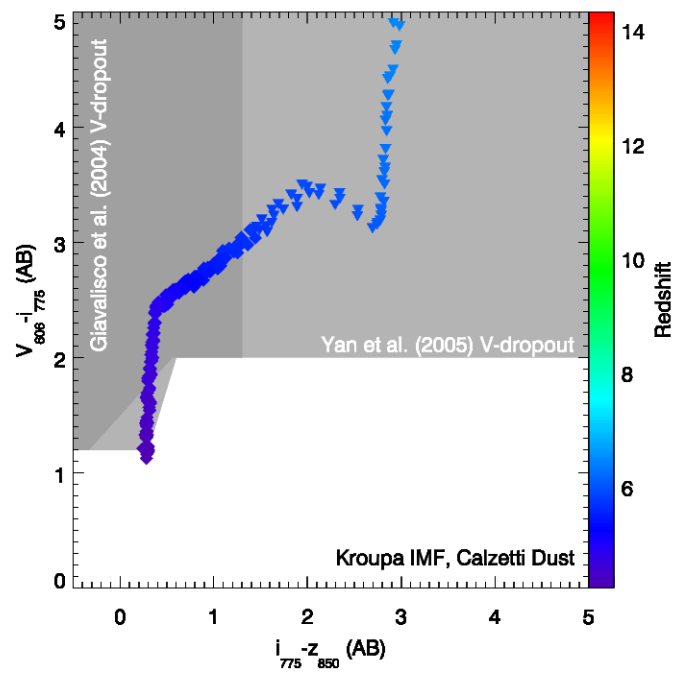

Fig. 4.- Calculated $V_{606}-i_{775}$ and $i_{775}-z_{850}$ colors with redshift for a simulated quasar host galaxy spectral energy distribution (SED). Shown is the location of the SED in this color-color space when the system SED has significant AGN activity (triangles, $5.7 \lesssim z \lesssim 6.7$ ) or is passively-evolving (diamonds, $z \lesssim 5.7$ ). A Also shown are the Giavalisco et al. (2004a) GOODS and Yan et al. (2005) HUDF color selection criteria for galaxies at $z \sim 5$. The simulated galaxy satisfies these criteria during its passive evolution phase. At higher redshifts, during the epoch of AGN activity, the system will move into $i_{775}$-dropout samples $\left(i_{775}-z_{850} \gtrsim 1.3\right.$, see Fig. 5 ).

criteria used in previous observational work. For convenience, the quasar host galaxy magnitudes in the salient observational filters at twenty redshifts $z=14-4.25$ are presented in Table 1 .

\section{1. $V_{606}$-dropout Selection}

The multi-color HST ACS observations in the GOODS fields allow for a variety of color-color selection criteria to identify LBG samples. The bluest color Lyman-break dropout techniques for HST data that overlap with the simulation redshift coverage are the $V_{606}$-dropout selections at $z \sim 5$. Giavalisco et al. (2004a) presented a $V_{606}$-dropout selection for GOODS by the color criterion

$$
\begin{gathered}
\left\{\left[\left(V_{606}-i_{775}\right)>1.5+0.9 \times\left(i_{775}-z_{850}\right)\right]\right. \\
\left.\vee\left[\left(V_{606}-i_{775}\right)>2.0\right]\right\} \\
\wedge \\
\left\{\left(V_{606}-i_{775}\right) \geq 1.2\right\} \\
\wedge \\
\left\{\left(i_{775}-z_{850}\right) \leq 1.3\right\},
\end{gathered}
$$

where $\vee$ and $\wedge$ represent the logical "OR" and "AND" operations, and used galaxies selected in this manner to estimate the global star formation rate density at $z \sim 5$. A similar selection criterion, defined as

$$
\begin{aligned}
\left\{\left[\left(V_{606}-i_{775}\right)>1.2\right]\right. & \left.\wedge\left[\left(i_{775}-z_{850}\right) \leq 0.3\right]\right\} \\
& \vee \\
\left\{\left[\left(V_{606}-i_{775}\right)>0.4\right.\right. & \left.+2.67\left(i_{775}-z_{850}\right)\right] \\
\wedge\left[0.3 \leq\left(i_{775}\right.\right. & \left.\left.\left.-z_{850}\right) \leq 0.6\right]\right\} \\
& \vee \\
\left\{\left[\left(V_{606}-i_{775}\right) \geq 2.0\right]\right. & \left.\wedge\left[\left(i_{775}-z_{850}\right)>0.6\right]\right\},
\end{aligned}
$$

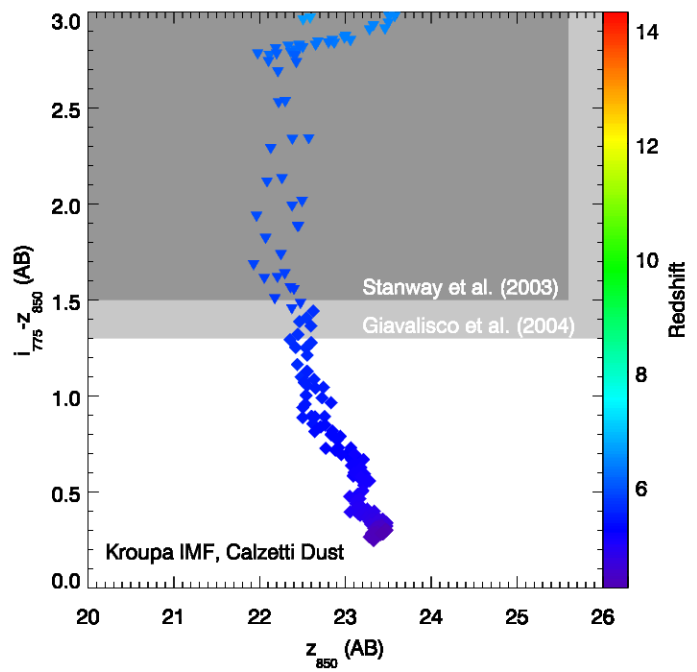

FIG. 5.- Calculated $i_{775}-z_{850}$ color and $z_{850}$ magnitude with redshift for a simulated quasar host galaxy spectral energy distribution (SED). Shown is the location of the SED in this color-color space when the system SED is roughly starburstdominated (circles, $z \gtrsim 6.7$ ), shows significant AGN activity (triangles, $5.7 \lesssim z \lesssim 6.7$ ) or is passively-evolving (diamonds, $z \lesssim 5.7$ ). Also shown are the Giavalisco et al. (2004a) and Stanway et al. (2003) GOODS color selection criteria for galaxies at $z \sim 6$. The simulated galaxy satisfies these criteria during the epoch of AGN activity and, owing to its activity and large stellar mass, is considerably brighter than the candidate $z \sim 6$ GOODS galaxies.

was used by Yan et al. (2005) to find $z \sim 5$ galaxies in the HUDF for examination with coincident Spitzer IRAC observations. The application of stellar population synthesis modeling found that galaxies at $z \sim 5$ in GOODS have typical stellar masses of $M_{\star} \sim 10^{10} M_{\odot}$, stellar ages of $t \sim 1 \mathrm{Gyr}$, low extinction, and a wide range of metallicities.

Figure 4 shows the calculated $V_{606}-i_{775}$ and $i_{775}-z_{850}$ colors for the massive host galaxy at redshifts $z>4$. The system satisfies the $V_{606}$-dropout color criterion over its entire passive-evolution phase at redshifts $z \lesssim 5.7$ when the SMBH accretion and star formation rates are low and the stellar component is passively aging. In $V_{606}$-dropout samples, the massive descendants of $z \sim 6$ quasars could appear as bright objects $\left(i_{775} \sim 23.5\right)$ with red $K_{s}-[3.6 \mu \mathrm{m}]$ colors (see Fig. 3 or Table 1). At higher redshifts $(z \gtrsim 5.7)$, during an epoch of AGN activity, the system will move into $i_{775}$ - and $i^{\prime}$-dropout samples $\left(i_{775}-z_{850} \gtrsim 1.3\right.$, see Fig. [5] and 4.2$)$.

\section{2. $i_{775}$-dropout Selection}

Earlier in the evolution of the quasar host galaxy, during its phase of significant AGN activity at times $5.7 \lesssim z \lesssim 6.7$, the IGM absorption has moved into the F775W filter and the system becomes red in the $i_{775}-z_{850}$ color. To identify $i_{775^{-}}$ dropouts at $z \sim 6$ in the GOODS ACS data, Giavalisco et al. (2004b) and Dickinson et al. (2004) used a simple criterion given by

$$
\left(i_{775}-z_{850}\right) \geq 1.3 .
$$

The GOODS data was also used to define a an $i_{775}$-dropout sample by Stanway et al. (2003), using similar criteria

$$
\begin{gathered}
\left\{z_{850}<25.6\right\} \\
\wedge \\
\left\{\left(i_{775}-z_{850}\right) \geq 1.5\right\} .
\end{gathered}
$$


TABLE 1

Photometric Evolution (AB Magnitudes)

\begin{tabular}{|c|c|c|c|c|c|c|c|c|c|c|c|c|c|}
\hline Redshift & $\mathrm{F} 435 \mathrm{~W}$ & F606W & $\mathrm{F} 775 \mathrm{~W}$ & $\mathrm{i}^{\prime}$ & $\overline{z^{\prime}}$ & F850LP & F110W & $\mathrm{F} 160 \mathrm{~W}$ & $\mathrm{~K}_{s}$ & {$[3.6 \mu m]$} & {$[4.5 \mu m]$} & {$[5.8 \mu m]$} & {$[8 \mu m]$} \\
\hline 14.00 & $>30$ & $>30$ & $>30$ & $>30$ & $>30$ & $>30$ & $>30$ & $>30$ & 26.11 & 26.27 & 26.39 & 26.40 & 26.40 \\
\hline 13.18 & $>30$ & $>30$ & $>30$ & $>30$ & $>30$ & $>30$ & $>30$ & 27.77 & 25.68 & 25.81 & 25.89 & 25.65 & 25.57 \\
\hline 12.32 & $>30$ & $>30$ & $>30$ & $>30$ & $>30$ & $>30$ & $>30$ & 24.94 & 23.97 & 24.09 & 24.16 & 23.80 & 23.81 \\
\hline 11.04 & $>30$ & $>30$ & $>30$ & $>30$ & $>30$ & $>30$ & $>30$ & 25.08 & 24.70 & 24.60 & 24.25 & 23.83 & 23.83 \\
\hline 10.03 & $>30$ & $>30$ & $>30$ & $>30$ & $>30$ & $>30$ & 27.08 & 23.61 & 23.56 & 23.47 & 22.92 & 22.79 & 22.74 \\
\hline 9.600 & $>30$ & $>30$ & $>30$ & $>30$ & $>30$ & $>30$ & 24.01 & 21.67 & 21.75 & 21.96 & 21.59 & 21.61 & 21.61 \\
\hline 9.209 & $>30$ & $>30$ & $>30$ & $>30$ & $>30$ & $>30$ & 23.83 & 21.98 & 21.98 & 22.03 & 21.57 & 21.57 & 21.48 \\
\hline 8.555 & $>30$ & $>30$ & $>30$ & $>30$ & 29.85 & 29.22 & 21.69 & 20.45 & 20.49 & 20.49 & 20.25 & 20.26 & 20.11 \\
\hline 7.970 & $>30$ & $>30$ & $>30$ & $>30$ & 27.92 & 27.84 & 21.93 & 20.92 & 20.92 & 20.30 & 20.08 & 20.05 & 19.97 \\
\hline 7.467 & $>30$ & $>30$ & $>30$ & $>30$ & 27.14 & 26.00 & 22.35 & 21.49 & 21.41 & 20.40 & 20.28 & 20.20 & 20.12 \\
\hline 7.008 & $>30$ & $>30$ & 27.74 & 27.45 & 24.58 & 24.04 & 21.96 & 21.32 & 21.12 & 20.07 & 19.96 & 19.81 & 19.80 \\
\hline 6.607 & $>30$ & $>30$ & 25.62 & 25.47 & 22.69 & 22.50 & 21.31 & 20.84 & 20.55 & 19.72 & 19.56 & 19.39 & 19.52 \\
\hline 6.467 & $>30$ & $>30$ & 26.69 & 26.55 & 23.74 & 23.57 & 22.38 & 21.84 & 21.51 & 20.18 & 20.06 & 19.99 & 19.94 \\
\hline 6.300 & $>30$ & 29.90 & 25.81 & 25.71 & 22.95 & 22.87 & 22.09 & 21.66 & 21.31 & 20.15 & 20.00 & 19.90 & 19.91 \\
\hline 5.993 & $>30$ & 27.99 & 24.94 & 24.75 & 22.24 & 22.22 & 21.89 & 21.58 & 21.21 & 20.16 & 19.96 & 19.87 & 19.94 \\
\hline 5.563 & $>30$ & 26.68 & 23.91 & 23.76 & 22.57 & 22.55 & 22.24 & 21.96 & 21.48 & 20.25 & 20.06 & 20.00 & 20.02 \\
\hline 5.007 & $>30$ & 26.22 & 23.80 & 23.69 & 23.21 & 23.19 & 22.87 & 22.40 & 21.58 & 20.23 & 20.10 & 20.03 & 20.02 \\
\hline 4.491 & $>30$ & 25.28 & 23.68 & 23.65 & 23.37 & 23.35 & 23.02 & 22.26 & 20.91 & 20.07 & 19.98 & 19.91 & 19.93 \\
\hline 4.250 & 29.51 & 24.99 & 23.79 & 23.77 & 23.49 & 23.47 & 23.12 & 22.22 & 20.77 & 20.06 & 19.96 & 19.89 & 19.90 \\
\hline
\end{tabular}

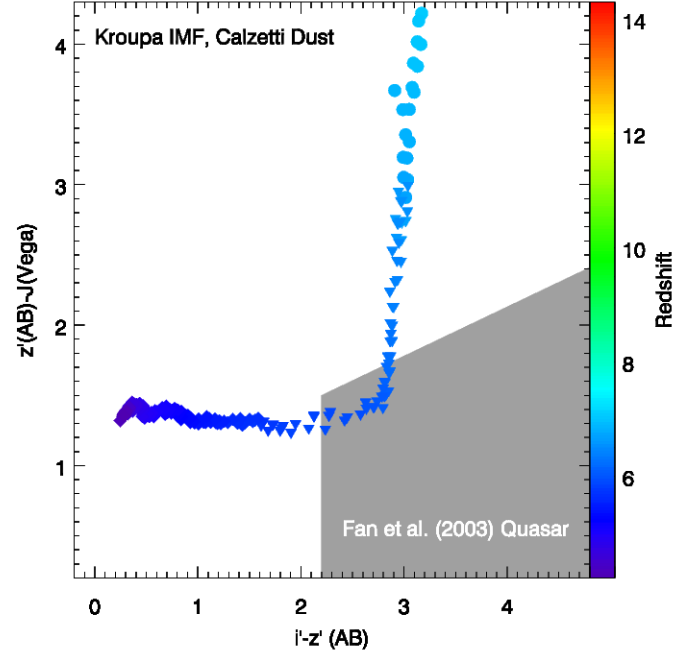

FIG. 6. - Calculated $z_{\mathrm{AB}}^{\prime}-J_{\mathrm{Vega}}$ and $i_{\mathrm{AB}}^{\prime}-z_{\mathrm{AB}}^{\prime}$ colors with redshift for a simulated quasar host galaxy spectral energy distribution (SED). Shown is the location of the SED in this color-color space when the system SED is roughly starburstdominated (circles, $z \gtrsim 6.7$ ), has significant AGN activity (triangles, $5.7 \lesssim z \lesssim 6.7$ ), or is passively-evolving (diamonds, $z \lesssim 5.7)$. Also shown is the SDSS color selection criterion for $z \approx 6$ quasars from Fan et al. (2003). The simulated galaxy satisfies the Fan et al. (2003) color criterion during its quasar phase near $z \sim 6$ and color-selected as a SDSS quasar candidate. However, the system is $\sim 2$ magnitudes fainter than the $z<20.2$ criterion used by Fan et al. (2003) to define their quasar sample.

(see also Bunker et al. 2004). Both samples were used to estimate the global star formation rate density at $z \sim 6$.

Figure 5 shows the $i_{775}-z_{850}$ color and $z_{850}$ magnitude of the quasar host galaxy during its evolution. The simulated galaxy satisfies these criteria during its phase of AGN activity and, owing to its activity and large stellar mass $\left(M_{\star}=1.64 \times\right.$ $10^{12} M_{\odot}$ at $\left.z=6.5\right)$, is considerably brighter $\left(\Delta z_{850} \sim 3\right)$ than candidate $z \sim 6$ GOODS galaxies $\left(z_{850} \sim 25.5\right.$, near the Stanway et al. (2003) magnitude cut).

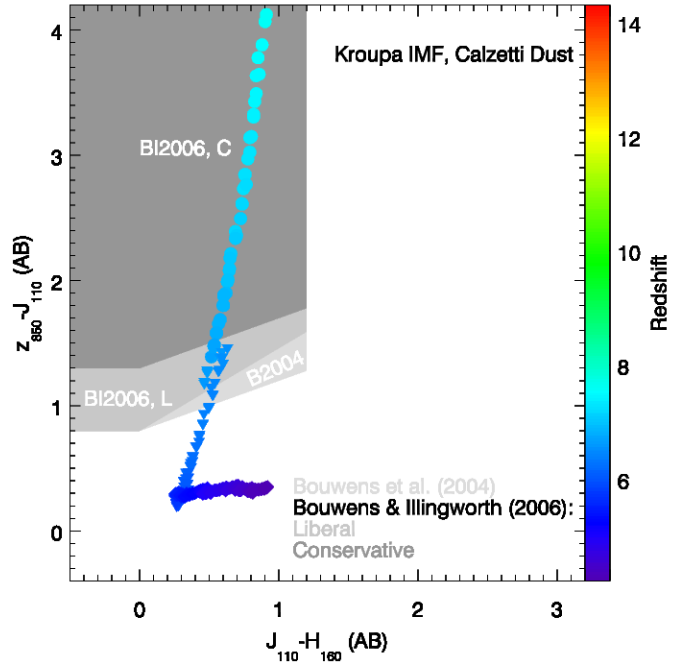

FIG. 7.- Calculated $z_{850}-J_{110}$ and $J_{110}-H_{160}$ colors with redshift for a simulated quasar host galaxy spectral energy distribution (SED). Shown is the location of the SED in this color-color space when the system SED is starburst-dominated (circles, $z \gtrsim 6.7$ ), shows significant AGN activity (triangles, $5.7 \lesssim z \lesssim 6.7$ ), or is passively-evolving (diamonds, $z<5.7$ ). Also shown are the Bouwens et al. (2004) (light gray), Bouwens \& Illingworth (2006)- "liberal" (gray), and Bouwens \& Illingworth (2006)"conservative" (dark gray) color selection criteria for galaxies at $z \gtrsim 7$ in NICMOS observations of the Hubble Ultra Deep Field (HUDF). The simulated $z \sim 6$ quasar host galaxy satisfies these criteria primarily during its starburst-dominated evolution phase at redshifts $z \sim 7-8$, but owing to its extreme star-formation rates and stellar mass it is typically $\sim 6$ F160W-magnitudes brighter than HUDF candidates.

\subsection{SDSS Quasar Selection}

The identification of quasars by multi-color selection criteria has been a common practice for nearly twenty years (e.g Warren et al. 1987; Irwin et al. 1991), and has proven especially fruitful for identifying distant $(z \sim 6)$ quasars in the SDSS (e.g., Fan et al. 2000, 2001a, 2003, 2006). The revised 
selection criterion used by Fan et al. (2003), given by

$$
\begin{gathered}
\left\{z^{\prime}<20.2\right\} \\
\wedge \\
\left\{\left(i^{\prime}-z^{\prime}\right)>2.2\right\} \\
\wedge \\
\left\{\left(z^{\prime}-J_{\text {Vega }}\right)<1.5+0.35\left(i^{\prime}-z^{\prime}-2.2\right)\right\},
\end{gathered}
$$

incorporates $i^{\prime}$-dropouts determined by the SDSS data with supplemental $J$-band photometry to distinguish from objects with red $z^{\prime}-J$ colors, such as cool stars. Figure 6 shows the $z_{\mathrm{AB}}^{\prime}-J_{\text {Vega }}$ and $i_{\mathrm{AB}}^{\prime}-z_{\mathrm{AB}}^{\prime}$ colors calculated for the quasar host galaxy over its evolution, including the phase of strong AGN activity at $z \sim 6.5$. The simulated galaxy satisfies the Fan et al. (2003) color criteria during its quasar phase near $z \sim 6.5$ and would be color-selected as a SDSS quasar candidate. However, the system is $\sim 2$ magnitudes fainter than the $z *<20.2$ criterion used by Fan et al. (2003) to define their quasar sample. The quasar phase in this simulation $(z \sim 6.5)$ occurs near the beginning of a broader phase of accretion and AGN activity $(7 \gtrsim z \gtrsim 5.5)$, before the SMBH mass reaches its final mass. Had the peak unobscured SMBH accretion occurred slightly later (e.g., $z \sim 5.8$ rather than $z \sim 6.5$ ), the system would have met the magnitude cut for the Fan et al. (2003) SDSS quasar selection. We note that at $z \gtrsim 6.3$ the system moves near the L-dwarf locus in the $z^{\prime}-J$ vs. $i^{\prime}-z^{\prime}$ color space, similar to the behavior of the quasar evolutionary track examined by Fan et al. (2003) to inform their choice of color selection criteria.

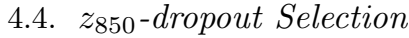

The combined ACS and NICMOS observations of the HUDF and GOODS fields have allowed for a search of $z \sim$ $7-8$ galaxies by looking for $z_{850}$-dropouts. Yan \& Windhorst (2004) found three $z_{850}$-dropout galaxies in the HUDF but concluded that their red $J_{110}-H_{160}$ colors made them unlikely to reside at $z \sim 7$. Bouwens et al. (2004) and Bouwens \& Illingworth (2006) used $z_{850}$-dropout criteria with a blue $J_{110}-H_{160}$ color cut to find $z \sim 7$ galaxies more robustly. These color-color criteria include the selection

$$
\begin{gathered}
\left\{\left(z_{850}-J_{110}\right)>0.8\right\} \\
\wedge \\
\left\{\left(z_{850}-J_{110}\right)>0.8+0.66\left(J_{110}-H_{160}\right)\right\} \\
\wedge \\
\left\{\left(J_{110}-H_{160}\right)<1.2\right\}
\end{gathered}
$$

from Bouwens et al. (2004), the "liberal" color selection from Bouwens \& Illingworth (2006)

$$
\begin{gathered}
\left\{\left(z_{850}-J_{110}\right)>0.8\right\} \\
\wedge \\
\left\{\left(z_{850}-J_{110}\right)>0.8+0.4\left(J_{110}-H_{160}\right)\right\} \\
\wedge \\
\left\{\left(J_{110}-H_{160}\right)<1.2\right\}
\end{gathered}
$$

and the Bouwens \& Illingworth (2006) "conservative" selection

$$
\begin{gathered}
\left\{\left(z_{850}-J_{110}\right)>1.3\right\} \\
\wedge \\
\left\{\left(z_{850}-J_{110}\right)>1.3+0.4\left(J_{110}-H_{160}\right)\right\} \\
\wedge \\
\left\{\left(J_{110}-H_{160}\right)<1.2\right\} .
\end{gathered}
$$

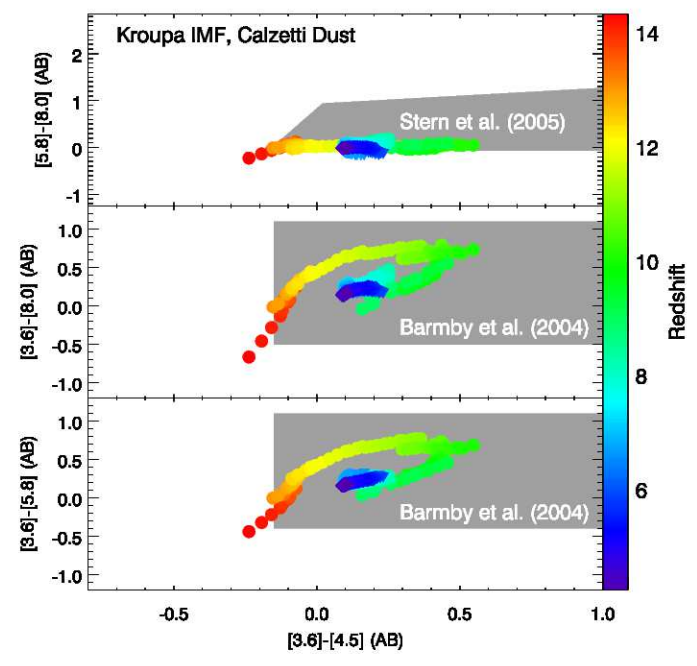

FIG. 8.- Calculated Infrared Array Camera (IRAC) colors with redshift for a simulated quasar host galaxy spectral energy distribution (SED). Shown is the location of the SED in this color-color space when the system SED is starburstdominated (circles, $z \gtrsim 6.7$ ), shows significant AGN activity (triangles, $5.7 \lesssim z \lesssim 6.7$ ) or is passively-evolving (diamonds, $z<5.7)$. Also shown are the AGES active galaxy IR-selection from Stern et al. (2005b) and the Barmby et al. (2004) IRAC color selection criteria for LBGs at $z \sim 3$. The simulated galaxy satisfies these criteria during most of its evolution and would be detected in both the Barmby et al. (2004) IRAC observations and the IRAC Shallow Survey (Eisenhardt et al. 2004), but is $3-4 \mathrm{AB}$ magnitudes fainter than the AGES sub-sample of the IRAC Shallow Survey.

These photometric selections find $<5$ high-redshift galaxies in GOODS, suggesting a rapid decline in the density of luminous star-forming galaxies and a decrease of the characteristic luminosity of galaxies by $\approx 1 \mathrm{AB}$ magnitude from $z \gtrsim 6$ to $z \sim 7-8$

Figure 7 shows the $z_{850}-J_{110}$ and $J_{110}-H_{160}$ colors calculated for the quasar host galaxy with redshift. The lowerredshift epochs that cover the AGN activity and passiveevolution phases occur below $z \sim 6.7$ and largely do not satisfy the $z_{850}$-dropout color criteria. The starburst phase at $z \gtrsim 7$, when the luminosity of the system is dominated by the young stars generated by the enormous star formation rates $\mathrm{SFR} \sim 10^{3}-10^{4}$, covers the entire $z_{850}-J_{110}$ color extent of the Bouwens \& Illingworth (2006) conservative selection. The starbursting quasar progenitor grows to $M_{\star} \approx 1.6 \times 10^{12} M_{\odot}$ in stars with a mass-weighted stellar age of $\langle t\rangle=250 \mathrm{Myr}$ at $z=7$, powering a remarkable luminosity $\sim 6 \mathrm{~F} 160 \mathrm{~W}$-magnitudes brighter than $z_{850}$-dropout $z \sim 7$ candidates in GOODS. If $z \sim 6$ quasars form in the manner suggested by the Li et al. (2006) simulations, a $z_{850}$-dropout or similar selection criterion would be well-matched to find quasar progenitors during the peak of their spheroid formation.

\subsection{IRAC-based Selection}

In surveys at redshifts $z \lesssim 4$, the IR properties of both AGN and galaxies have been used to design color-color selection criteria using IRAC bands. Stern et al. (2005a) showed that the $[3.6 \mu \mathrm{m}]-[4.5 \mu \mathrm{m}]$ color of broad-line AGN at $1 \lesssim z \lesssim 4$ are typically redder than galaxies at $z \lesssim 2$. Their selection, given by,

$$
\{([5.8 \mu \mathrm{m}]-[8.0 \mu \mathrm{m}])>-0.07\}
$$




$$
\wedge
$$

$$
\begin{gathered}
\{([3.6 \mu \mathrm{m}]-[4.5 \mu \mathrm{m}])>-0.157 \\
\quad+0.188([5.8 \mu \mathrm{m}]-[8.0 \mu \mathrm{m}])\} \\
\wedge \\
\{([3.6 \mu \mathrm{m}]-[4.5 \mu \mathrm{m}])>-2.77 \\
\quad+2.97([5.8 \mu \mathrm{m}]-[8.0 \mu \mathrm{m}])\}
\end{gathered}
$$

identified $90 \%$ of the spectroscopically identified type 1 AGN in the AGN and Galaxy Evolution Survey (AGES).

Barmby et al. (2004) used template SEDs to estimate the location of galaxies at $z \gtrsim 2$ in the $[3.6 \mu \mathrm{m}]-[5.8 \mu \mathrm{m}]$ vs. $[3.6 \mu \mathrm{m}]-[4.5 \mu \mathrm{m}]$ and $[3.6 \mu \mathrm{m}]-[8.0 \mu \mathrm{m}]$ vs. $[3.6 \mu \mathrm{m}]-[4.5 \mu \mathrm{m}]$ color-color spaces. Comparing with a sample of confirmed $z \sim 3$ LBG galaxies, they found that the majority of the $\mathrm{LBGs}$ resided in the IRAC color-color regions

$$
\begin{gathered}
\{([3.6 \mu \mathrm{m}]-[4.5 \mu \mathrm{m}])>-0.15\} \\
\wedge \\
\{1.1>([3.6 \mu \mathrm{m}]-[5.8 \mu \mathrm{m}])>-0.4\}
\end{gathered}
$$

and

$$
\begin{gathered}
\{([3.6 \mu \mathrm{m}]-[4.5 \mu \mathrm{m}])>-0.15\} \\
\wedge \\
\{1.1>([3.6 \mu \mathrm{m}]-[8.0 \mu \mathrm{m}])>-0.5\} .
\end{gathered}
$$

Barmby et al. (2004) combined these criteria with selections based on $R_{s}$-band magnitude. At the redshifts we consider, the quasar host galaxy always has significant IGM attenuation of the $R_{s}$-band and is significantly redder in $R_{s}-[3.6 \mu \mathrm{m}]$ color than the galaxies examined by Barmby et al. (2004). We therefore compare only with their IRAC color-color selections.

Figure 8 shows the calculated IRAC colors for the simulated quasar host galaxy at redshifts $z>4$. The galaxy satisfies the Barmby et al. (2004) and Stern et al. (2005b) criteria during most of its evolution. The $[3.6 \mu \mathrm{m}]-[4.5 \mu \mathrm{m}]$ color begins blue at redshifts $z \gtrsim 12$ when the star-forming SED has its rest-frame near-UV and optical emission in the shortestwavelength IRAC bands. As the redshift decreases and the stellar populations age, the $[3.6 \mu \mathrm{m}]-[4.5 \mu \mathrm{m}]$ color increases until the Balmer $/ 4000 \AA$ break passes through these bands and the galaxy becomes very red in $[3.6 \mu \mathrm{m}]-[4.5 \mu \mathrm{m}]$. At lower redshifts, the system becomes relatively stable in its $[3.6 \mu \mathrm{m}]-[4.5 \mu \mathrm{m}]$ color for the remainder of the simulation. The system has a consistently flat $[5.8 \mu \mathrm{m}]-[8.0 \mu \mathrm{m}]$ color, and would allow for its selection by the Stern et al. (2005b) criteria. However, the AGES sub-sample of the IRAC Shallow Survey (Eisenhardt et al. 2004) has a limiting magnitude $\sim 3$ magnitudes fainter than the complete Shallow Survey in the $[3.6 \mu \mathrm{m}]$-band, and is too shallow to detect the simulated quasar host galaxy. The Barmby et al. (2004) color selections involve the $[3.6 \mu \mathrm{m}]$-band and have considerably more evolution than redder bands since the $[3.6 \mu \mathrm{m}]$-band transitions through the Balmer $/ 4000 \AA$ break (see $\$ 4.6$ below). The large color-color area of the Barmby et al. (2004) criterion would allow for the quasar host galaxy to be selected over almost the entire duration of the simulation (redshifts $z \lesssim 12$ ). All three IRAC criteria examined would be convenient methods to define color selected samples that could include quasar host galaxies at $z \gtrsim 4$.

\subsection{High-Redshift 4000A/Balmer Breaks}

The presence of $4000 \AA$ or Balmer breaks in the observed SEDs of galaxies has been cited as evidence for evolved stellar populations in galaxies at $z \gtrsim 2$ (e.g. Franx et al. 2003). The calculated quasar progenitor star formation history (Fig. 1)

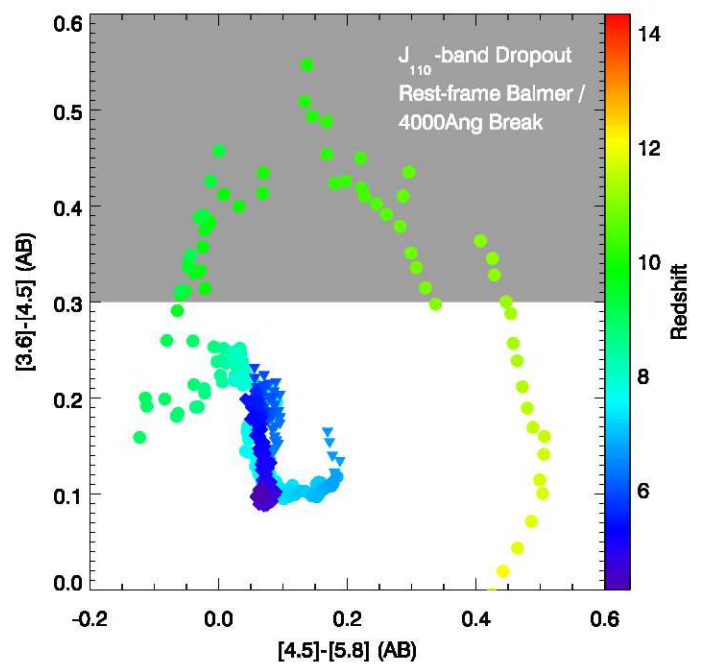

Fig. 9. - Calculated Infrared Array Camera (IRAC) $[3.6 \mu \mathrm{m}]-[4.5 \mu \mathrm{m}]$ vs. $[4.5 \mu \mathrm{m}]-[5.8 \mu \mathrm{m}]$ colors with redshift for a simulated quasar host galaxy spectral energy distribution (SED). Shown is the location of the SED in this colorcolor space when the system SED is starburst-dominated (circles, $z \gtrsim 6.7$ ), shows significant AGN activity (triangles, $5.7 \lesssim z \lesssim 6.7$ ) or is passively-evolving (diamonds, $z<5.7$ ). At $z \sim 10$, the quasar progenitor has already been forming stars for $\gtrsim 200 \mathrm{Myr}$ and will display a rest-frame Balmer/4000A spectral break. The break could be observed as a red $[3.6 \mu \mathrm{m}]-[4.5 \mu \mathrm{m}]$ color in $J_{110}$-dropout samples, analogous to the $K_{s}-[3.6 \mu \mathrm{m}]$ spectral break measured in $z_{850^{-}}$ band dropouts at lower redshifts (e.g., Labbé et al. 2006).

suggests that a significant population of $\operatorname{stars}\left(M_{\star} \approx 10^{11} M_{\odot}\right)$ can form in such systems by $z \sim 12$. As these stars age they can contribute a small $4000 \AA$ / Balmer spectral break in the galaxy SED, as is apparent in the $z \sim 10$ SED in Figure 3 and may be detected photometrically in the near-IR.

Figure 9 shows the calculated IRAC $[3.6 \mu \mathrm{m}]-[4.5 \mu \mathrm{m}]$ vs. $[4.5 \mu \mathrm{m}]-[5.8 \mu \mathrm{m}]$ colors for the simulated quasar host galaxy at redshifts $z>4$. The observable near-IR SED is very flat over the majority of the galaxy's evolution, remaining at $([4.5 \mu \mathrm{m}]-[5.8 \mu \mathrm{m}])<0.2$ and $([4.5 \mu \mathrm{m}]-[5.8 \mu \mathrm{m}])<$ 0.27 at redshifts $z<9$. At higher redshifts $z \sim 9-11$ when the SED would move into $J_{110}$-dropout samples, the Balmer $/ 4000 \AA$ break moves into the $[3.6 \mu \mathrm{m}]$-band and causes the $[3.6 \mu \mathrm{m}]-[4.5 \mu \mathrm{m}]$ color to redder by $\sim 0.2-0.3$ magnitudes. As the Balmer/4000 $\AA$ break moves through the $[4.5 \mu \mathrm{m}]$-band, the evolutionary track of the SED reverses direction at higher redshifts and the system becomes substantially bluer in $[3.6 \mu \mathrm{m}]-[4.5 \mu \mathrm{m}]$ as the filters begin to probe the region of the SED sensitive to the large star formation rate (at $z \sim 12$, see the upper left panel of Fig. 3). In contrast, the trajectory of local galaxy SEDs with mature stellar populations in color-spaces sensitive to optical breaks continue to redden as the SEDs are shifted to higher redshifts (e.g., $J_{s}-K$ color at redshifts $z>2.5$, see Figure 1 of Franx et al. [2003). The extremum of the $[3.6 \mu \mathrm{m}]-[4.5 \mu \mathrm{m}]$ at $z \sim 9-11$ owing to the rest-frame optical break makes the color a useful indicator for the presence of mature stars in $J_{110}$-dropout samples.

A completely analogous behavior can occur in lower redshift samples in bluer bands. SED fits to $z_{850}$-dropout galaxies in the HUDF at $z \sim 7$ show evidence for a rest-frame optical break in their $K-[3.6 \mu \mathrm{m}]$ colors (Labbé et al. 2006), as do $z \approx 6$ galaxies (Yan et al. 2005; Evles et al. 2005), 


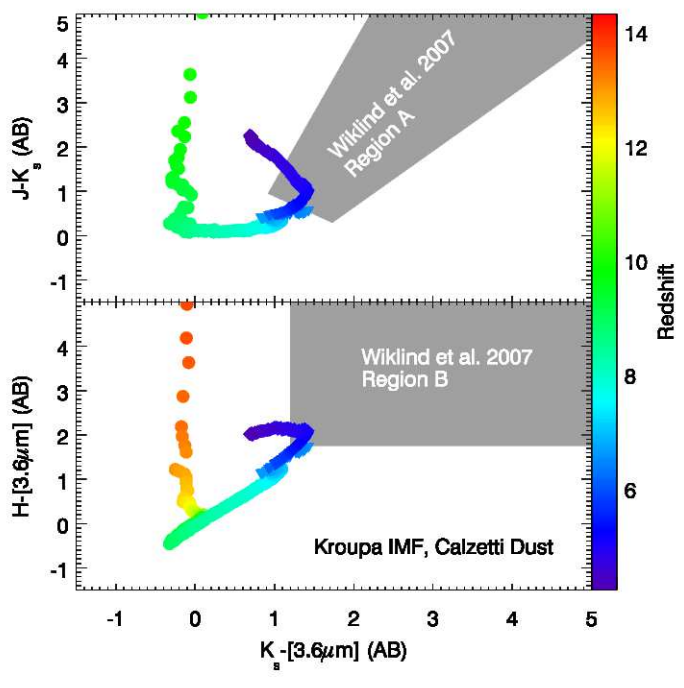

Fig. 10.- Calculated near-infrared $J, H, K_{s}$, and Infrared Array Camera (IRAC) $[3.6 \mu \mathrm{m}]$ colors with redshift for a simulated quasar host galaxy spectral energy distribution (SED). Shown is the location of the SED in this color-color space when the system SED is starburst-dominated (circles, $z \gtrsim$ 6.7), shows significant AGN activity (triangles, $5.7 \lesssim z \lesssim 6.7$ ) or is passively-evolving (diamonds, $z<5.7$ ). Also shown are the "Region A" and "Region B" color selection criteria from Wiklind et al. (2007a, b $)$ designed to identify poststarburst systems at $z \sim 5$ via the Balmer $/ 4000 \AA$ break. The quasar descendant satisfies the Wiklind et al. (2007a b) criteria over the redshift range $4.8 \lesssim z \lesssim 5.5$, during the poststarburst, passively-evolving phase of the system's evolution. The Wiklind et al. (2007a b) criteria are also briefly satisfied by the galaxy at $z \sim 6.5$ during the height of the quasar activity.

and possibly indicate an evolved stellar component. Recently, Wiklind et al. (2007a,b) have proposed Balmer break selection criteria for post-starburst systems at $z \sim 5$ using VLT/ISAAC near-IR $J, H$, and $K_{s}$ and IRAC $[3.6 \mu \mathrm{m}]$ band observations of the GOODS fields. Their selection is defined by a union of objects in two regions: a "Region A" in the $J-K_{s}$ vs. $H-[3.6 \mu \mathrm{m}]$ color space, given by

$$
\begin{gathered}
\left\{\left(J-K_{s}\right)<-1.94+3.14\left(K_{s}-[3.6 \mu \mathrm{m}]\right)\right\} \\
\wedge \\
\left\{\left(J-K_{s}\right)>-1.90+1.27\left(K_{s}-[3.6 \mu \mathrm{m}]\right)\right\} \\
\left.\left.\hat{A}-K_{s}\right)>1.71-0.82\left(K_{s}-[3.6 \mu \mathrm{m}]\right)\right\},
\end{gathered}
$$

and a "Region B" in the $H-[3.6 \mu \mathrm{m}]$ vs. $H-[3.6 \mu \mathrm{m}]$ color space, given by

$$
\begin{gathered}
\{(H-[3.6 \mu \mathrm{m}])>1.75\} \\
\wedge \\
\left\{\left(K_{s}-[3.6 \mu \mathrm{m}]\right)>1.20\right\} .
\end{gathered}
$$

Figure 10 shows the calculated near-infrared $J, H, K_{s}$, and IRAC $[3.6 \mu \mathrm{m}]$ colors with redshift for the quasar host galaxy SED in relation to Regions A and B from Wiklind et al. (2007a b). The quasar descendant satisfies the Wiklind et al. (2007a b) criteria over the redshift range $4.8 \lesssim z \lesssim 5.5$, during the post-starburst, passively-evolving phase of the system's evolution. The comparison suggests that the Wiklind et al. (2007a, b) criteria would select the descendants of $z \sim 6$ quasars during the passively-evolving phase near redshift $z \sim 5$. While the Wiklind et al. (2007a, b) selection was designed to find massive $\left(M_{\star} \sim 10^{11} M_{\odot}\right)$ and evolved galaxies near $z \sim 5$, the agreement may be somewhat surprising given that the evolution of the simulated quasar host SED in the $J_{-}, H_{-}, K_{s^{-}}$, and [3.6 $\left.\mu \mathrm{m}\right]$-bands is qualitatively different than the evolving SED models used to design the Wiklind et al. $(2007 \mathrm{a}, \mathrm{b})$ selection. However, we note that over the range of redshifts where the quasar host galaxy would be detectable in the GOODS and ISAAC data the simulated system resides in the locus of observed galaxies in the Wiklind et al. $(2007 \mathrm{a}, \mathrm{b})$ sample in the $\left(J-K_{s}\right)-(H-[3.6 \mu \mathrm{m}])-\left(K_{s}-[3.6 \mu \mathrm{m}]\right)$ color space. We also note that the Wiklind et al. (2007a b) criteria are briefly satisfied by the galaxy at $z \sim 6.5$ during the height of the quasar activity, but such bright sources can be discriminated from inactive high-redshift galaxies through spectroscopic observations.

\section{OBSERVABILITY}

The comparisons between the redshift-dependent photometric properties of massive quasar host galaxies and color selection criteria in 4.14.6 demonstrate that the SEDs of the most massive high-redshift galaxies likely satisfy a variety of existing photometric selection techniques. However, these high-redshift galaxies are extremely massive and therefore extraordinarily rare. To determine whether existing or future galaxy surveys would include such rare systems in their samples, the observability of these massive galaxies must be estimated. A straightforward quantification of the observability is to calculate the fractional sky coverage and flux limit required to include and detect some defined galaxy sample. Below, we use the evolution of the quasar host galaxy SED, the $\Lambda$ CDM cosmological model, and Press \& Schechter (1974) theory to estimate these quantities.

The comoving volume element $\mathrm{d} V$ of a spherical redshift shell of thickness $\mathrm{d} z$ at comoving distance $r(z)$ and redshift $z$ can be written

$$
\mathrm{d} V=4 \pi r^{2}(z) \frac{\mathrm{d} r}{\mathrm{~d} z} \mathrm{~d} z
$$

where

$$
\begin{gathered}
r(z)=\int_{0}^{z} \frac{\mathrm{d} r}{\mathrm{~d} z^{\prime}} \mathrm{d} z^{\prime} \\
=\int_{0}^{z} \frac{c \mathrm{~d} z^{\prime}}{H\left(z^{\prime}\right)},
\end{gathered}
$$

$c$ is the speed of light, the redshift-dependent Hubble parameter for a flat universe is

$$
H(z)=H_{0}\left[\Omega_{\mathrm{M}}(1+z)^{3}+\Omega_{\Lambda}\right]^{1 / 2},
$$

and $H_{0}$ is the Hubble constant today. The number of galaxies $\mathrm{d} N$ in this comoving volume element is then simply $\mathrm{d} N=n(z) \mathrm{d} V$, where $n(z)$ is the redshift-dependent comoving number density of galaxies that comprise the sample. For the form of $n(z)$, we use the mass function proposed by Sheth \& Tormen (1999) to extend the Press \& Schechter (1974) theory of halo formation to model ellipsoidal collapse. Assuming an overdensity threshold $\delta_{c}=1.686$ for halo collapse, we can define the quantity $\nu=\sqrt{a} \delta_{c} /[\sigma(M) D(z)]$ that relates the root-mean-squared fluctuations $\sigma(M)$ on a mass scale $M$, the linear growth factor

$$
D(z)=D_{0} H(z) \int_{z}^{\infty} \frac{\left(1+z^{\prime}\right) \mathrm{d} z^{\prime}}{H^{3}\left(z^{\prime}\right)}
$$

with $D_{0}$ normalized such that $D(z=0)=1$, and $\delta_{c}$ to describe the rarity of the density peak that collapsed to form the halo of mass $M$. Here the constant $a=0.707$ was determined from cosmological N-body simulations by Sheth \& Tormen 
(1999). In terms of the peak rarity $\nu$, the comoving number density of galaxies above some given mass $M_{\text {low }}$ is then

$$
\begin{gathered}
n\left(M_{\text {low }}, z\right)=\int_{\ln M_{\text {low }}}^{\infty} \frac{\mathrm{d} n}{\mathrm{~d} \ln m} \mathrm{~d} \ln m \\
=\Omega_{m} \rho_{c} \int_{\ln M_{\text {low }}}^{\infty} \frac{A}{m \sigma} \sqrt{\frac{2}{\pi}}\left(1+\nu^{-2 p}\right) \nu e^{-\frac{\nu^{2}}{2}} \\
\times \frac{\mathrm{d} \sigma}{\mathrm{d} \ln m} \mathrm{~d} \ln m,
\end{gathered}
$$

where the parameter $A=0.322$ is constrained such that $\int_{0}^{\infty}(\mathrm{d} n / \mathrm{d} \ln m) \mathrm{d} \ln m=1$ and $p=0.3$ is determined from fitting to halo mass functions measured in cosmological simulations. If we choose $M_{\text {low }}$ to correspond to the redshiftdependent virial mass of a quasar host galaxy and assume that more massive halos will host brighter galaxies, then $M_{\text {low }}$ defines a luminosity-selected sample of very massive galaxies at redshift $z$ with comoving number density $n(z)$.

To calculate an actual number $N$ of galaxies, a redshift interval $z_{\text {low }} \leq z \leq z_{\text {high }}$ must be prescribed to define the comoving volume that hosts the sample as

$$
\begin{gathered}
N\left(z_{\text {low }}, z_{\text {high }}, M_{\text {low }}\right)=\int n\left(M_{\text {low }}, z\right) \mathrm{d} V \\
\quad=4 \pi c \int_{z_{\text {low }}}^{z_{\text {high }}} \frac{n\left(M_{\text {low }}, z^{\prime}\right) r^{2}\left(z^{\prime}\right) \mathrm{d} z^{\prime}}{H\left(z^{\prime}\right)} .
\end{gathered}
$$

For a given selection criterion, such as a Lyman-break dropout, $z_{\text {low }}$ will correspond to the redshift at which galaxy SEDs satisfy the color criteria. At $z_{\text {low }}$ the least massive galaxy in the sample will have a magnitude $m_{\mathrm{AB}}$ that corresponds to the brightest possible magnitude limit of a survey that could detect galaxies with mass $M_{\text {low }}$. Typically, for dropout criteria this magnitude limit would apply to the redder of the two bands (e.g., $i_{775}$-band for $V_{606}$-dropouts). However, such a survey would have a very small comoving volume (since $z_{\text {low }}=z_{\text {high }}$ in this case) and it is desirable to improve the magnitude limit to increase the comoving volume of the survey. As the magnitude limit of the survey is improved to a new $m_{\mathrm{AB}}^{\prime}, z_{\text {high }}$ increases to the epoch when galaxies with mass $M_{\text {low }}$ first became brighter than $m_{\mathrm{AB}}^{\prime}$. For a given photometric selection which sets $z_{\text {low }}$, and survey magnitude limit $m_{\mathrm{AB}}$, the fractional sky coverage needed to detect $N_{d}$ galaxies with mass greater than $M_{\text {low }}$ at $z_{\text {low }}$ is

$$
f=N_{d} / N\left(z_{\text {low }}, z_{\text {high }}\left[m_{\mathrm{AB}}\right], M_{\text {low }}\right)
$$

While this is conceptually straightforward, there are two immediate complications. First, the correspondence between the upper redshift limit $z_{\text {high }}$ and the magnitude limit $m_{\mathrm{AB}}$ depends on the redshift-dependent photometric properties of galaxies with mass $M_{\text {low }}$. Second, galaxies with mass $M_{\text {low }}$ at epoch $z_{\text {low }}$ are evolving and will have some lower mass at higher redshifts. Fortunately, the calculations of the redshiftdependent galaxy SED described in $\$ 3$ provide the desired $z_{\text {high }}-m_{\mathrm{AB}}$ correspondence. For the mass accretion history the merger-tree from the Li et al. (2006) simulation is boxcar averaged with window width of $\mathrm{d} z=0.3$. The resulting smooth mass accretion history increases rapidly from $z \sim 14$ to $z \sim 9$ owing to frequent mergers, and has a shape similar to the Wechsler et al. (2002) form for dark matter halo mass accretion histories. We note the observability calculation is insensitive to the exact shape of the mass accretion history as long as the rapid decline in virial mass at $z>9$ is reproduced.

The relation between fractional sky coverage and survey limiting magnitude is plotted in Figure 11 for a variety of color selection criteria explored in 3 including $V_{606}$-dropout (Eq. 5. $\left.z_{\text {low }} \sim 4.5\right), i_{775}$-dropout (Eq. 7] $\left.z_{\text {low }} \sim 5.7\right), z_{850^{-}}$ dropout (Eq. 12, $\left.z_{\text {low }} \sim 6.8\right)$, and $[3.6 \mu \mathrm{m}]-[4.5 \mu \mathrm{m}]>0.3$ break $\left(z_{\text {low }} \sim 9.1\right)$ samples. For these color selection criteria, the quasar host galaxy virial mass in the Li et al. (2006) simulation increases from $M_{\text {low }}=5.22 \times 10^{12} h^{-1} M_{\odot}$ at $z_{\text {low }}=9.1$ to $M_{\text {low }}=8.01 \times 10^{12} h^{-1} M_{\odot}$ at $z_{\text {low }} \sim 4.5$. Beginning with the brightest possible magnitude limit, corresponding to the luminosity of $M_{\text {low }}$-mass galaxies at $z_{\text {low }}$, the necessary fractional sky coverage declines as the sample comoving volume increases with limiting magnitude until $z_{\text {high }}$ is reached. At larger (fainter) magnitudes, the fractional sky coverage becomes flat. The width of the transition region before the fractional sky coverage flattens is set by the redshift range over which the luminosity declines. For the $V_{606^{-}}, i_{775^{-}}$, and $z_{850^{-}}$ dropout samples, IGM absorption sets $z_{\text {high }}$ to approximately the redshift when Ly $\alpha$ moves into the $i_{775^{-}}, z_{850^{-}}$, and $J_{110^{-}}$ bands, respectively. The Lyman break is dramatic at such high redshifts and, when combined with the decrease in $\mathrm{d} r / \mathrm{d} z$ and $n(z)$ with redshift, causes the fractional sky coverage to flatten quickly beyond the brightest limiting magnitude. The larger transition width for the $z_{850}$-dropout selection relative to the bluer color selections is set by the comparably large bandwidth of the F110W filter. For the $[3.6 \mu \mathrm{m}]-[4.5 \mu \mathrm{m}]-$ break selection, the fractional sky coverage artificially flattens at the $[4.5 \mu \mathrm{m}]$-band magnitude of the system at $z \sim 14$ $([4.5 \mu \mathrm{m}] \sim 26)$, where the simulation begins. Since these galaxies are bright $([4.5 \mu \mathrm{m}]<24$ to $z \sim 12)$, only a relatively small fractional sky coverage is needed to detect them. However, given the decline of $\mathrm{d} r / \mathrm{d} z$ and $n(z)$ with redshift, such a sample would be dominated by systems at $z \sim 4$.

Given the rapid decline of the comoving number density $n$ with mass $M_{\text {low }}$ at fixed redshift, a useful comparison is the fractional sky coverage needed to observe systems that are less massive than the Li et al. (2006) simulated galaxy by a factor of $\sim 2$. Figure 11 also shows the require fractional sky coverage for these systems, assuming their photometric evolution is similar to massive galaxies and their luminosity scales with their halo mass. Such systems are clearly $\sim 10 \times$ more abundant than galaxies twice their mass. These systems would have stellar masses of $7 \times 10^{11} M_{\odot}$, and SMBH masses $M_{\mathrm{BH}} \sim 10^{9} M_{\odot}$, and could host quasars at $z \lesssim 6$. The virial mass of such galaxies is similar to that inferred from the clustering of quasars at in the 2dF, SDSS, and other surveys (Porciani et al. 2004; Porciani \& Norberg 2006; Wake et al. 2004; Croom et al. 2005; Coil et al. 2006; Shen et al. 2007) and measurements of the quasar proximity effect (Faucher-Giguere et al. 2007; Kim \& Croft 2007; Guimaraes et al. 2007), and are qualitatively similar to the system simulated by Li et al. (2006) and the large set of $z \sim 6$ merger simulations performed by Robertson et al. (2006b). The detection of the host galaxies of such quasars in Lymanbreak dropout or Balmer-break samples would also provide interesting insights into the formation of massive high-redshift galaxies (for a possible sample of such systems at $z \sim 5$, see Wiklind et al. 2007a, b).

For comparison, we also show the necessary sky coverage to find objects with an abundance fixed to the comoving number density $\left(n \sim 4 \times 10^{-9} h^{-3} \mathrm{Mpc}^{-3}\right)$ of halos with virial mass $M_{\text {vir }} \gtrsim 8 \times 10^{12} h^{-1} M_{\odot}$ at $z \sim 6.5$ if their luminosity evolution is similar to the simulated quasar host galaxy (Fig. 11, dashed-dotted lines). Correspondingly, such systems have an abundance similar to massive quasar host galaxies when they fall in $i_{775}$-dropout samples. For higher-redshift selections, this estimate of the massive galaxy abundance is more favorable for finding the progenitors of $z \sim 6$ quasars and is comparable to our $M_{\text {low }}=0.5 M_{\text {vir }}$ abundance estimates.

The estimated survey parameters for finding high-redshift massive galaxies can be compared with the properties of existing and future photometric surveys to gauge the likelihood for the most massive high-redshift galaxies to be found in actual observational samples. The largest existing survey is the SDSS, which has magnitude limits of $i^{\prime} \sim 22.3$ and $z^{\prime} \sim 20.8$ (York et al. 2000) over $8452 \mathrm{deg}^{2}{ }^{11}$. While the SDSS has suf-

11 http://www.sdss.org/status/ 


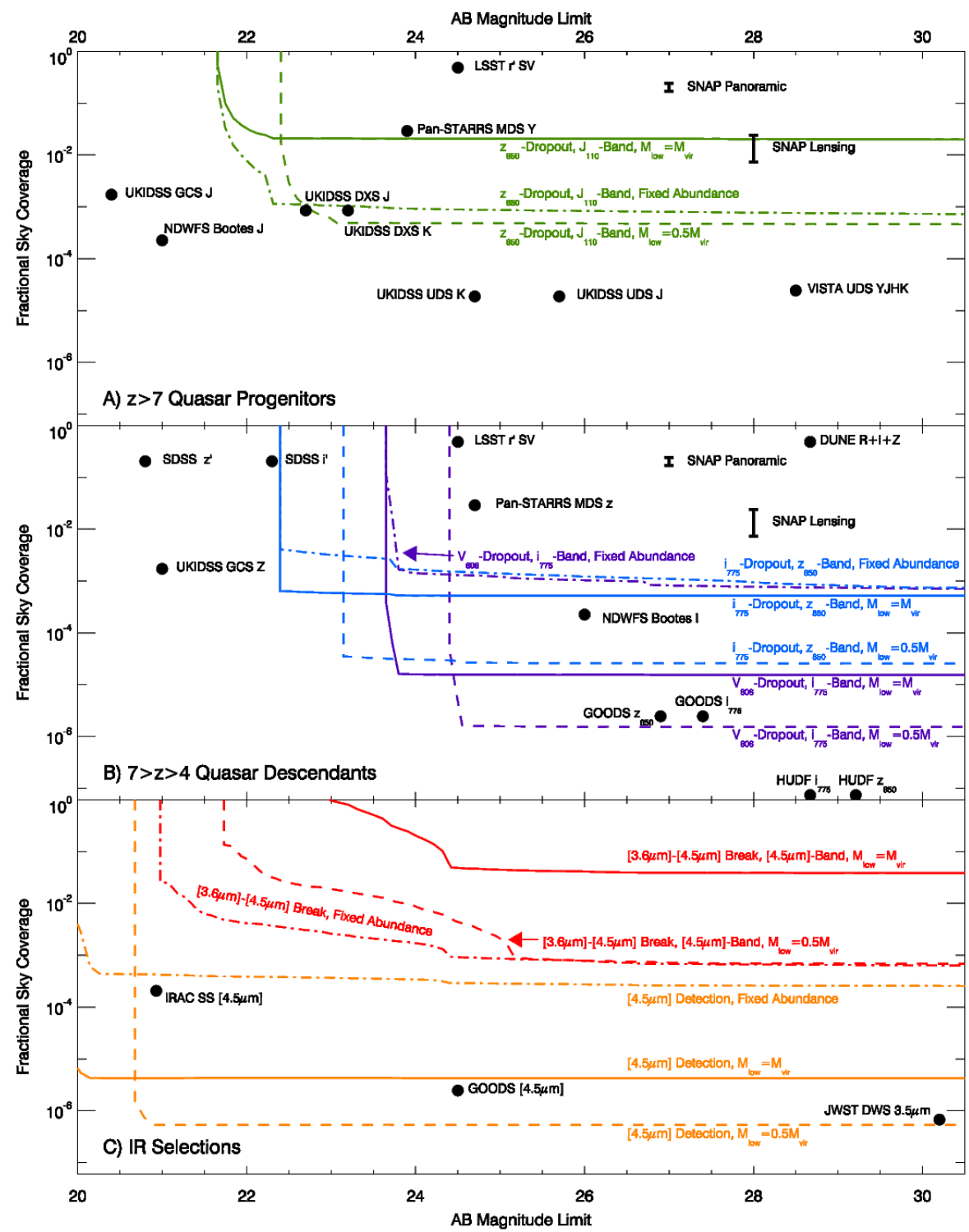

FIG. 11.- Estimated survey parameters required to find $z \gtrsim 7$ quasar progenitors (upper panel) and quasar descendants at redshifts $7 \gtrsim z \gtrsim 4$ (middle panel), or detect quasar host galaxies in the near infrared (bottom panel). Shown is the fractional sky coverage and minimum AB magnitude limit needed to build $V_{606}$-dropout (purple), $i 775$-dropout (blue), $z_{850}$-dropout (green), and $[3.6 \mu \mathrm{m}]-[4.5 \mu \mathrm{m}]$ break (red) samples that include a galaxy more massive than the virial mass $M_{\text {vir }}$ (solid line) or $0.5 M_{\text {vir }}$ of halos more massive than the simulated galaxy at $z \sim 6.5$ (dashed-dotted line) is also shown. As the dropout selection moves to redder bands and higher redshifts, the comoving volume and redshift interval over which massive galaxies satisfy selection criteria decreases. The comoving number density of massive galaxies, calculated using the Sheth \& Tormen (1999) mass function, also declines rapidly at high-redshifts. The combination these effects requires large fractional sky coverage to find starbursting quasar progenitors at $z \geq 7$ (e.g. $z 850$-dropout or $[3.6 \mu \mathrm{m}]-[4.5 \mu \mathrm{m}]$ break selections). The circles show the parameters of the existing Hubble UDF (i $775-$ and $z 850^{-b a n d}$ ), GOODS (i775- $z 850-$, and $[4.5 \mu \mathrm{m}]-\mathrm{b} a n d s)$, SDSS $\left(i^{\prime}-\right.$ and $z^{\prime}$-bands), NOAO Wide Deep Field Survey ( $I$ - and $J$-bands), UKIRT Deep Sky Survey ( $Z$ - and $J-$ bands for the GOODS $\left(i 775^{-}, z 850^{-}\right.$, and $[4.5 \mu \mathrm{m}]$-bands), SDSS $\left(i-\right.$ and $z^{\prime}$-bands), NOAO Wide Deep Field Survey ( $I$ - and $J$-bands), UKIRT Deep Sky Survey ( $Z$ - and $J$ - bands for the from the Dark Universe Explorer $(R+I+Z$ band) and the Ultra-deep Visible and Infrared Telescope for Astronomy survey ( $Y$-, J-, $H-$, and $K$-bands) could detect $z \sim 5-6$. Even fire wider future surveys with redder sensitivity, such as the Pan-STARS Medium Deep Survey ( $z$ - and $Y$-band), Large Synoptic Survey Telescope observations ( $r$ - Single Visit), Webb Space Telescope Deep-Wide Survey [Gardner et al, 2006) would need to be considerably wider to find the $z>7$ starbursting progenitors of $z \sim 6$ quasars. Also shown is

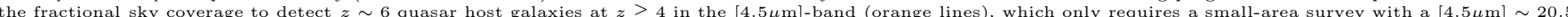
magnitude limit.

ficient area to capture the most massive galaxies at redshifts as high as $z \gtrsim 12$, the bright magnitude limits and lack of infrared coverage makes the detection of extremely massive high-redshift $(z>6)$ galaxies in the SDSS implausible. Of course, as has been beautifully demonstrated, the rarer subpopulation of massive galaxies in a bright quasar phase has been detected in $\sim 1 / 2$ the total SDSS area (e.g., Fan et al. 2003) as the remarkable luminosity of the $z \sim 6$ quasar sample and the large sky coverage of the SDSS allows for the detection of tens of systems.
Compared with SDSS, deeper but smaller surveys have more advantageous parameters for finding Lyman-break dropouts at redshifts $z \gtrsim 4.5$. For instance, the Boötes field of the National Optical Astronomy Observatory Wide Deep Field Survey (Jannuzi \& Dey 1999) has an $9.3 \mathrm{deg}^{2}$ field with magnitude limits of $I=26$ and $J=21$. While the $J$-band coverage is likely too shallow to detect highredshift quasar host galaxies, this area-magnitude combination should be sufficient to find the most massive galaxies at $z \sim 4.5-5$ in $V$-dropout samples. The accompany- 
ing IRAC Shallow Survey (Eisenhardt et al. 2004), with 8.5 $\operatorname{deg}^{2}$ coverage down to $[4.5 \mu \mathrm{m}] \approx 20.9$ would detect these systems as well as higher-redshift $I$-dropouts in the NOAO NWDFS (for galaxies with photometric redshifts $z \lesssim 2$, see Brodwin et al. 2006). The United Kingdom Infrared Telescope Deep Sky Survey (UKIDSS, Warren \& Hewett 2002; Warren et al. 2007) includes a Galaxy Cluster Survey (GCS, $70.9 \mathrm{deg}^{2}$ area to $Z \approx 21$ and $J \approx 20.4$ ), a Deep Extragalactic Survey (DXS, $35 \mathrm{deg}^{2}$ area to $J \approx 23.2$ and $K=22.7$ ), and an Ultradeep Survey (UDS, $0.77 \mathrm{deg}^{2}$ area to $J \approx 25.7$ and $K \approx 24.7$, see Lawrence et al. 2006). While the UKIDSS GCS is too shallow to find the very massive galaxies we model, the UKIDSS DXS and UDS could detect such systems in the $J$ and $K$-bands at $z \gtrsim 4$. The UKIDSS DXS may even have enough area to detect massive quasar progenitors at $z \gtrsim 7$ (see also Warren \& Hewett 2002), which would be an exciting prospect, but the lack of deep coverage at shorter wavelengths in the DXS may limit its ability to identify such systems as Lyman-break dropouts. A proposed UDS with the Visible and Infrared Survey Telescope for Astronomy (VISTA) could reach 5- $\sigma \mathrm{AB}$ limiting magnitudes of $Y=26.7, J=26.6$, $H=26.1$, and $K_{s}=25.6$ over $\mathrm{a} \sim 1 \mathrm{deg}^{2}$ field (M. Franx, private communication). While the proposed VISTA observations would be significantly deeper than the UKDISS UDS or the NDWFS, the size of the field could limit the survey to finding $z \sim 6$ quasar host galaxies at $z \lesssim 5$.

The GOODS observations that cover $365 \mathrm{arcmin}^{2}$ reach depths of $i_{775} \approx 27.4$ and $z_{850} \approx 26.9$ with ACS (Giavalisco et al. 2004b) and $[4.5 \mu \mathrm{m}]=24.5$ with IRAC (Dickinson et al. 2003). The GOODS $V_{606}$-dropout sample has the necessary sky coverage to include a $M_{\star} \sim 5 \times 10^{11} M_{\odot}$ stellar mass galaxy at $z \sim 4-5$ with $i_{775} \sim 25$ and detections in all redder bands, though we are not aware of any such systems in the current GOODS data. Less massive $\left(M_{\star} \sim\right.$ $\left.10^{11} M_{\odot}\right)$ systems are much more abundant and our estimate would predict a handful of such $z \sim 5$ systems in GOODS, which may have already been detected (e.g., Stark et al. 2006). The HUDF data, with an $11 \operatorname{arcmin}^{2}$ area and magnitude limits of $i_{775}=29.21$ and $z_{850}=28.67$ (Beckwith et al. 2006), is simply too narrow to include objects as rare as $z \sim 6$ quasar host galaxies. We note briefly that these simulations suggest that to detect $M_{\star} \sim 5 \times 10^{11} M_{\odot}$ galaxies in $J_{110^{-}}$ dropout samples at $z \gtrsim 7$ (e.g. Mobasher et al. 2005, see also Chen \& Marzke (2004) and Dunlop et al. (2006)), a survey 3-4 orders of magnitude larger than the HUDF would be necessary.

Future surveys with wide area coverage and deep imaging have a substantially better opportunity to detect the starbursting progenitors of $z \sim 6$ quasars. The PanSTARRS project is planning a $1200 \mathrm{deg}^{2}$ Medium Deep Survey with magnitude limits of $z_{0.89 \mu \mathrm{m}}=24.7$ and $Y_{1.02 \mu \mathrm{m}}=$ $23.9^{12}$, which could find massive quasar progenitors in $z$ band dropout samples at $z \gtrsim 7$. The Large Synoptic Survey Telescope would make short work of finding the most massive high-redshift galaxies if its $Y$-band Single Visit Depth is comparable to its target $r=24.5$ depth $^{13}$. The Supernovae Acceleration Probe (SNAP) is planning a weak lensing survey of $300-1000 \mathrm{deg}^{2}$ to $m_{\mathrm{AB}}=28$ and a wide-area $\left(7000-10000 \mathrm{deg}^{2}\right)$ survey with a depth of $m_{\mathrm{AB}}=27^{14}$. If a near-IR $\mathrm{HgCdTe}$ device flies with the mission and reaches similar sensitivities down to $\lambda \sim 1.5 \mu \mathrm{m}$ over the same area, SNAP could detect the progenitors of $z \sim 6$ quasars at redshifts approaching $z \sim 10$. The Dark Universe Explorer mission will survey $20,000 \mathrm{deg}^{2}$ with a composite $R+I+Z$-band

\footnotetext{
12 http://pan-starrs.ifa.hawaii.edu/

13 http://www.lsst.org/Science/docs/SRD_summary.pdf

14 http://snap.lbl.gov
}

in the red optical and will reach an effective $I \sim 24.5$ limiting magnitude (Réfrégier et al. 2006), which should detect massive spheroids at $z \sim 5$. The James Webb Space Telescope easily has enough sensitivity to detect massive quasar progenitors out to $z \gtrsim 15$ (with stellar masses $M_{\star} \lesssim 10^{10} M_{\odot}$ ), but currently suggested observations, such as a Deep-Wide Survey with a $100 \operatorname{arcmin}^{2}$ area and limiting magnitude of $m_{\mathrm{AB}} \approx 30.2$ at $\lambda=3.5 \mu \mathrm{m}$ (Gardner et al. 2006), would be too narrow to find such rare objects at the highest redshifts.

\section{DISCUSSION}

The discovery of very massive high-redshift galaxies would provide an interesting new perspective on the structure formation process. While the larger abundance of less-massive galaxies allow the redshift evolution of such systems to be researched by characterizing the statistical properties of populations of objects, the rarity of the most massive galaxies will likely limit our ability to draw inferences about their formation from population studies. If the most massive galaxies at the current epoch were a largely heterogeneous population, their rarity could prove a significant limitation in unravelling the various modes for their formation.

Fortunately, the most massive galaxies today are a roughly homogeneous population. Studies of the color bimodality of galaxies (e.g., Strateva et al. 2001; Bell et al. 2004; Baldry et al. 2004) show that the most luminous galaxies are almost uniformly red $\left(g^{\prime}-r^{\prime} \gtrsim 1.2\right)$. Such galaxies populate the center of galaxy clusters and their properties are roughly uniform between clusters at the same epoch (e.g., Bower et al. 1992; Gladders \& Yee 2000). Importantly, these massive galaxies have been demonstrated to contain supermassive black holes with mass $M_{\mathrm{BH}} \gtrsim 10^{9} M_{\odot}$ (i.e. M87, Harms et al. 1994; Macchetto et al. 1997). The large SMBH masses directly connect the population of the most massive galaxies at low redshift to the luminous quasars at $z \sim 6$, since $10^{9} M_{\odot}$ SMBHs are the most credible engine to power $z \sim 6$ quasars, and suggest that the most luminous highredshift quasars evolve into a roughly homogeneous population at the current epoch. Our calculations support this possibility, under the condition that the most massive galaxies undergo little star formation and their stellar populations evolve passively from $z \sim 4$ to the present. Observed cluster ellipticals have stellar formation epochs of at least $z \gtrsim 2$ (e.g., van Dokkum \& Franx 2001; Gebhardt et al. 2003). We note that this picture does not preclude future major mergers between spheroids at lower redshifts (e.g., van Dokkum 2005; Bell et al. 2006), which may have some relation to core-cusp bimodality (e.g., Faber et al. 1997; Lauer et al. 2006, Kormendy et al., in preparation, Krause et al., in preparation, though see Ferrarese et al. (2006)) that is the primary heterogeneity in massive galaxies. Even so, this bimodality exists primarily in systems with $M_{V}>-22$, with more luminous systems mostly displaying surface brightness cores.

The connection between the most massive galaxies in the present epoch and quasars at $z \sim 6$, forged by their shared SMBH masses, and the uniformity of the most massive local galaxies permits an argument for a single evolutionary scenario for the formation of the most massive galaxies from very high-redshifts to the present day. The appeal of a single mode of formation for the most massive galaxies stems primarily from the difficulty in forming luminous quasars at high-redshifts with $\gtrsim 10^{9} M_{\odot}$ SMBHs, the problem popularized by Efstathiou \& Rees (1988). Finding a robust way of growing SMBHs quickly in the limited time available before $z \sim 6$ is problematic, but the success of the Li et al. (2006) simulation of $z \sim 6$ quasar in producing a $\mathrm{SMBH}$ with a mass of $M_{\mathrm{BH}}>10^{9} M_{\odot}$ has provided some hope that highredshift quasars can be explained naturally in the context of the formation of rare density peaks in our $\Lambda \mathrm{CDM}$ cos- 
mology. The calculations performed in this paper provide a detailed characterization of the observable ramifications of this scenario, the foremost being the possible detection of the star-bursting progenitors of $z \sim 6$ quasars at higher redshifts $(z \gtrsim 6)$ with massive stellar populations $\left(M_{\star} \sim 10^{11.5-12.0}\right)$ in wide-area, Lyman-break dropout samples or through widearea IR searches for systems with rest-frame optical breaks at $z \sim 9$ and stellar masses of $M_{\star} \sim 10^{11} M_{\odot}$.

\section{CONCLUSIONS}

Combining hydrodynamical simulations of the hierarchical formation of a $z \sim 6$ quasar (Li et al. 2006), stellar population synthesis models (Leitherer et al. 1999; Vázquez \& Leitherer 2005), AGN spectral templates (Vanden Berk et al. 2001; Marconi et al. 2004), models for the wavelength-dependent attenuation owing to interstellar and intergalactic absorption (Calzetti et al. 1994, 2000; Madau 1995), and the transmissivity of telescopes, filters, and detectors, the photometric properties of a massive $z \sim 6$ quasar host galaxy are calculated at redshifts $z \sim 4-14$. The photometric properties of the quasar host galaxy reflect three main evolutionary phases in its formation; a starburst-dominated phase at $z \gtrsim 6.7$, an AGN phase at $5.7 \lesssim z \lesssim 6.7$ that includes optical quasar activity at $z \sim 6.5$, and a passive-evolution phase at $z \lesssim 5.7$. The photometric properties of the system are then compared with color-selection techniques for finding high-redshift galaxies. The main findings of these calculations follow.

- At very high-redshifts $z \sim 6.7-14$, multiple gas-rich mergers gives rise to star formation rates of SFR $10^{3}-10^{4}$, building a stellar spheroid of $>10^{12} M_{\odot}$ by $z \sim 7$ (Li et al. 2006). $)$ Our calculations show that these star formation rates naturally give rise to a photometric starburst phase with strong rest-frame UV and blue optical emission that satisfy HST ACS/NICMOS Lymanbreak criteria for galaxies at $z>7$ (e.g. Bouwens et al. 2004; Bouwens \& Illingworth 2006). Over this epoch, the near-infrared luminosity of the system peaks at $z \sim 8.5$ with $J_{110} \sim 21.7, H_{160} \sim 20.5$, and $K_{s} \sim 20.5$ in $\mathrm{AB}$ magnitudes.

- During the phase of AGN activity $(5.7 \lesssim z \lesssim 6.7)$, IGM absorption blue-ward of Ly $\alpha$ causes the system to satisfy both F775W-band dropout criteria for LBGs in GOODS (e.g. Stanwav et al. 2003; Giavalisco et al. 2004a) and the SDSS $i^{\prime}$-band dropout selection for high-redshift quasars (e.g. Fan et al. 2003). At higher redshifts IGM absorption begins to attenuate the $z^{\prime}$ band flux and push the quasar host galaxy towards the locus of L-dwarfs in the $z^{\prime}-J_{\text {Vega }}$ vs. $i^{\prime}-z^{\prime}$ color-color space. During this phase, the quasar host galaxy is usually $\sim 2 z^{\prime}$-band magnitudes fainter than the Fan et al. (2003) sample selection flux limit.

- At lower redshifts $(z \lesssim 5.7)$ after the starburst and AGN activity has declined, the quasar host galaxy sat- isfies $V_{606}$-dropout criteria for galaxies at $z \sim 5$ (e.g., Giavalisco et al. 2004b; Yan et al. 2005). The quasar descendant also satisfies Balmer break selection techniques designed to identify massive and evolved galaxies at $z \sim 5$ (e.g., Wiklind et al. 2007a b).

- Throughout the photometric evolution of the quasar host galaxy, the system satisfies Spitzer IRAC colorcolor selections for AGN (Stern et al. 2005b) and highredshift galaxies (Barmby et al. 2004).

- The area and depth necessary to find the most massive high-redshift galaxies in photometric surveys is estimated from our photometric modeling and the redshift-dependent abundance of dark matter halos (Press \& Schechter 1974; Sheth \& Tormen 1999). Existing surveys, such as the IRAC Shallow Survey or NOAO Deep-Wide Field Survey, may be large enough to find the massive $z \sim 4$ descendants of $z \sim 6$ quasars. Surveys of significant fractions of the sky ( $\gtrsim 0.001)$ in the $z_{850}$ and $J_{110}$ or similar bands, such as the UKIRT Deep Sky Survey, will be necessary to find the starbursting progenitors of $z \sim 6$ at high-redshifts $(z>7)$. Future surveys, such as Pan-STARRS, the Large Synoptic Survey Telescope, or the Supernovae Acceleration Probe, should have the necessary area and depth to find the most massive high-redshift galaxies.

- If future surveys contain a $J_{110}$-dropout massive quasar progenitor at $z \sim 10$, evidence for star formation at $z \gtrsim 12$ in the form of a Balmer/4000 break could be detected in the $[3.6 \mu \mathrm{m}]-[4.5 \mu \mathrm{m}]$ vs. $[4.5 \mu \mathrm{m}]-[5.8 \mu \mathrm{m}]$ color-color space.

The remarkable luminosity and early-formation time of the massive progenitor galaxies of $z \sim 6$ quasars could enable their detection at very high-redshifts $z \gtrsim 7$. The detection of these massive galaxies would help settle outstanding questions about how the rarest structures form in the $\Lambda$ CDM cosmology and what evolutionary processes give rise to $z \sim 6$ quasars. As observations must ultimately determine the existence and properties of the most massive high-redshift galaxies, we eagerly anticipate future efforts to detect and characterize these extraordinary quasar progenitors.

BER gratefully acknowledges support from a Spitzer Fellowship, through a NASA grant administered by the Spitzer Science Center, and thanks Hsiao-Wen Chen, Tiziana Di Matteo, Volker Springel, Marijn Franx, and Andrew Zentner for helpful comments. BER also thanks Dr. Wiklind for early access to his results before publication. This work was supported in part by NSF grants ACI 96-19019, AST 00-71019, AST 02-06299, and AST 03-07690, and NASA ATP grants NAG5-12140, NAG5-13292, and NAG5-13381. The simulations were performed at the Center for Parallel Astrophysical Computing at Harvard-Smithsonian Center for Astrophysics.

\section{REFERENCES}

Abel, T., Bryan, G. L., \& Norman, M. L. 2002, Science, 295, 93

Baldry, I. K., Glazebrook, K., Brinkmann, J., Ivezić, Ž., Lupton,

R. H., Nichol, R. C., \& Szalay, A. S. 2004, ApJ, 600, 681

Barmby, P., et al. 2004, ApJS, 154, 97

Barnes, J. E., \& Hernquist, L. 1996, ApJ, 471, 115

Barnes, J. E., \& Hernquist, L. E. 1991, ApJ, 370, L65

Barth, A. J., Ho, L. C., \& Sargent, W. L. W. 2003, ApJ, 583, 134

Barton, E. J., Davé, R., Smith, J.-D. T., Papovich, C., Hernquist, L., \& Springel, V. 2004, ApJ, 604, L1

Baugh, C. M., Cole, S., Frenk, C. S., \& Lacey, C. G. 1998, ApJ, 498, 504

Beckwith, S. V. W., et al. 2006, AJ, 132, 1729

Bell, E. F., et al. 2006, ApJ, 640, 241
—. 2004, ApJ, 608, 752

Bershady, M. A., Charlton, J. C., \& Geoffroy, J. M. 1999, ApJ, 518,103

Bertoldi, F., Carilli, C. L., Cox, P., Fan, X., Strauss, M. A., Beelen, A., Omont, A., \& Zylka, R. 2003, A\&A, 406, L55

Bohlin, R. C., Savage, B. D., \& Drake, J. F. 1978, ApJ, 224, 132

Bondi, H. 1952, MNRAS, 112, 195

Bouwens, R. J., \& Illingworth, G. D. 2006, Nature, 443, 189

Bouwens, R. J., et al. 2003, ApJ, 595, 589

-. 2004, ApJ, 616, L79

Bower, R. G., Lucey, J. R., \& Ellis, R. S. 1992, MNRAS, 254, 601

Bremer, M. N., Jensen, J. B., Lehnert, M. D., Schreiber, N. M. F., \& Douglas, L. 2004, ApJ, 615, L1 
Brodwin, M., et al. 2006, ApJ, 651, 791

Bromm, V., \& Larson, R. B. 2004, ARA\&A, 42, 79

Bullock, J. S., Kolatt, T. S., Sigad, Y., Somerville, R. S., Kravtsov, A. V., Klypin, A. A., Primack, J. R., \& Dekel, A. 2001, MNRAS, 321, 559

Bunker, A. J., Stanway, E. R., Ellis, R. S., \& McMahon, R. G. 2004, MNRAS, 355, 374

Bunker, A. J., Stanway, E. R., Ellis, R. S., McMahon, R. G., \& McCarthy, P. J. 2003, MNRAS, 342, L47

Calzetti, D. 1997, AJ, 113, 162

Calzetti, D., Armus, L., Bohlin, R. C., Kinney, A. L., Koornneef, J., \& Storchi-Bergmann, T. 2000, ApJ, 533, 682

Calzetti, D., Kinney, A. L., \& Storchi-Bergmann, T. 1994, ApJ, 429,582

Cardelli, J. A., Clayton, G. C., \& Mathis, J. S. 1989, ApJ, 345, 245

Carilli, C. L., et al. 2004, AJ, 128, 997

Chary, R.-R., Stern, D., \& Eisenhardt, P. 2005, ApJ, 635, L5

Chen, H.-W., \& Marzke, R. O. 2004, ApJ, 615, 603

Cohen, M., Wheaton, W. A., \& Megeath, S. T. 2003, AJ, 126, 1090

Coil, A. L., Newman, J. A., Cooper, M. C., Davis, M., Faber, S. M., Koo, D. C., \& Willmer, C. N. A. 2006, ApJ, 644, 671

Cowie, L. L., \& Hu, E. M. 1998, AJ, 115, 1319

Cox, A. N. 2000, Allen's astrophysical quantities (Allen's astrophysical quantities, 4th ed. Publisher: New York: AIP Press; Springer, 2000. Editedy by Arthur N. Cox. ISBN: 0387987460)

Cox, T. J., Di Matteo, T., Hernquist, L., Hopkins, P. F., Robertson, B., \& Springel, V. 2006a, ApJ, 643, 692

Cox, T. J., Dutta, S. N., Di Matteo, T., Hernquist, L., Hopkins, P. F., Robertson, B., \& Springel, V. 2006b, ApJ, 650, 791

Croom, S. M., et al. 2005, MNRAS, 356, 415

Daddi, E., et al. 2003, ApJ, 588, 50

Davé, R., Finlator, K., \& Oppenheimer, B. D. 2006, MNRAS, 370,273

Davé, R., Hernquist, L., Katz, N., \& Weinberg, D. H. 1999, ApJ, 511,521

Di Matteo, T., Springel, V., \& Hernquist, L. 2005, Nature, 433, 604

Dickinson, M., Giavalisco, M., \& The Goods Team. 2003, in The Mass of Galaxies at Low and High Redshift, ed. R. Bender \& A. Renzini, 324-+

Dickinson, M., et al. 2004, ApJ, 600, L99

Dunlop, J. S., Cirasuolo, M., \& McLure, R. J. 2006, ArXiv Astrophysics e-prints

Efstathiou, G., \& Rees, M. J. 1988, MNRAS, 230, 5P

Egami, E., et al. 2005, ApJ, 618, L5

Eisenhardt, P. R., et al. 2004, ApJS, 154, 48

Ellis, R., Santos, M. R., Kneib, J.-P., \& Kuijken, K. 2001, ApJ, 560, L119

Eyles, L., Bunker, A., Ellis, R., Lacy, M., Stanway, E., Stark, D., \& Chiu, K. 2006, ArXiv Astrophysics e-prints

Eyles, L. P., Bunker, A. J., Stanway, E. R., Lacy, M., Ellis, R. S., \& Doherty, M. 2005, MNRAS, 364, 443

Faber, S. M., et al. 1997, AJ, 114, 1771

Fabian, A. C. 1999, MNRAS, 308, L39

Fagotto, F., Bressan, A., Bertelli, G., \& Chiosi, C. 1994, A\&AS, 105,29

Fan, X., et al. 2004, AJ, 128, 515

—. 2001a, AJ, 122, 2833

-. 2006, AJ, 131, 1203

-. 2001b, AJ, 121, 31

-. 2003, AJ, 125,1649

-. 2000, AJ, 120, 1167

Faucher-Giguere, C.-A., Lidz, A., Zaldarraiga, M., \& Hernquist, L. 2007, ArXiv Astrophysics e-prints

Fazio, G. G., et al. 2004, ApJS, 154, 10

Ferrarese, L., et al. 2006, ApJS, 164, 334

Ferrarese, L., \& Merritt, D. 2000, ApJ, 539, L9

Finlator, K., Davé, R., Papovich, C., \& Hernquist, L. 2006, ApJ, 639,672

Fitzpatrick, E. L. 1986, AJ, 92, 1068

Ford, H. C., et al. 1998, in Proc. SPIE Vol. 3356, p. 234-248, Space Telescopes and Instruments V, ed. P. Y. Bely \& J. B. Breckinridge

Franx, M., et al. 2003, ApJ, 587, L79
Fukugita, M., Ichikawa, T., Gunn, J. E., Doi, M., Shimasaku, K., \& Schneider, D. P. 1996, AJ, 111, 1748

Gao, L., White, S. D. M., Jenkins, A., Frenk, C. S., \& Springel, V. 2005, MNRAS, 363, 379

Gardner, J. P., et al. 2006, Space Science Reviews, 123, 485

Gebhardt, K., et al. 2000, ApJ, 539, L13

-. 2003, ApJ, 597, 239

Genzel, R., et al. 1998, ApJ, 498, 579

Giavalisco, M., et al. 2004a, ApJ, 600, L103

-. 2004b, ApJ, 600, L93

Gladders, M. D., \& Yee, H. K. C. 2000, AJ, 120, 2148

Gordon, K. D., Clayton, G. C., Misselt, K. A., Landolt, A. U., \& Wolff, M. J. 2003, ApJ, 594, 279

Granato, G. L., De Zotti, G., Silva, L., Bressan, A., \& Danese, L. 2004, ApJ, 600, 580

Grazian, A., et al. 2006, A\&A, 449, 951

Guimaraes, R. N., Petitjean, P., Rolline, E. B., De Carvalho, R. R., Djorgovski, G., Srianand, R., Aghaee, A., \& Castro, S. 2007, ArXiv Astrophysics e-prints

Harford, A. G., \& Gnedin, N. Y. 2003, ApJ, 597, 74

Häring, N., \& Rix, H.-W. 2004, ApJ, 604, L89

Harms, R. J., et al. 1994, ApJ, 435, L35

Hernquist, L. 1989, Nature, 340, 687

Hopkins, P. F., Hernquist, L., Cox, T. J., Di Matteo, T., Robertson, B., \& Springel, V. 2006a, ApJS, 163, 1

Hopkins, P. F., Hernquist, L., Cox, T. J., Robertson, B., \& Krause, E. 2007a, ArXiv Astrophysics e-prints

Hopkins, P. F., Hernquist, L., Cox, T. J., Robertson, B., \& Springel, V. 2006b, ApJS, 163, 50

Hopkins, P. F., Hernquist, L., Martini, P., Cox, T. J., Robertson, B., Di Matteo, T., \& Springel, V. 2005, ApJ, 625, L71

Hopkins, P. F., Lidz, A., Hernquist, L., Coil, A. L., Myers, A. D., Cox, T. J., \& Spergel, D. N. 2006c, ArXiv Astrophysics e-prints

Hopkins, P. F., Richards, G. T., \& Hernquist, L. 2007b, ApJ, 654, 731

Hu, E. M., \& Cowie, L. L. 2006, Nature, 440, 1145

Hu, E. M., Cowie, L. L., McMahon, R. G., Capak, P., Iwamuro, F., Kneib, J.-P., Maihara, T., \& Motohara, K. 2002, ApJ, 568, L75

Irwin, M., McMahon, R. G., \& Hazard, C. 1991, in ASP Conf.

Ser. 21: The Space Distribution of Quasars, ed. D. Crampton, $117-126$

Iye, M., et al. 2006, Nature, 443, 186

Jannuzi, B. T., \& Dey, A. 1999, in ASP Conf. Ser. 191:

Photometric Redshifts and the Detection of High Redshift

Galaxies, ed. R. Weymann, L. Storrie-Lombardi, M. Sawicki, \&

R. Brunner, 111-+

Kashikawa, N., et al. 2006, ApJ, 648, 7

Katz, N., Hernquist, L., \& Weinberg, D. H. 1992, ApJ, 399, L109

Kauffmann, G., Colberg, J. M., Diaferio, A., \& White, S. D. M. 1999, MNRAS, 303, 188

Kauffmann, G., \& Haehnelt, M. 2000, MNRAS, 311, 576

Kennicutt, R. C. 1998, ApJ, 498, 541

Kim, Y.-R., \& Croft, R. 2007, ArXiv Astrophysics e-prints

King, A. 2003, ApJ, 596, L27

Kodaira, K., et al. 2003, PASJ, 55, L17

Koester, B. P., et al. 2007, ArXiv Astrophysics e-prints

Kormendy, J., \& Richstone, D. 1995, ARA\&A, 33, 581

Kroupa, P. 2001, MNRAS, 322, 231

-. 2002, Science, 295, 82

Kurk, J. D., Cimatti, A., di Serego Alighieri, S., Vernet, J.,

Daddi, E., Ferrara, A., \& Ciardi, B. 2004, A\&A, 422, L13

Labbé, I., Bouwens, R., Illingworth, G. D., \& Franx, M. 2006, ApJ, 649, L67

Labbé, I., et al. 2005, ApJ, 624, L81

Lanzetta, K. M., Yahil, A., \& Fernandez-Soto, A. 1996, Nature, 381,759

Larson, R. B., \& Tinsley, B. M. 1978, ApJ, 219, 46

Lauer, T. R., et al. 2006, ArXiv Astrophysics e-prints

Lawrence, A., et al. 2006, ArXiv Astrophysics e-prints

Lee, K.-S., Giavalisco, M., Gnedin, O. Y., Somerville, R. S.,

Ferguson, H. C., Dickinson, M., \& Ouchi, M. 2006, ApJ, 642, 63

Lehnert, M. D., \& Bremer, M. 2003, ApJ, 593, 630

Leitherer, C., et al. 1999, ApJS, 123, 3

Li, Y., et al. 2006, ArXiv Astrophysics e-prints

Lynden-Bell, D. 1969, Nature, 223, 690 
Macchetto, F., Marconi, A., Axon, D. J., Capetti, A., Sparks, W., \& Crane, P. 1997, ApJ, 489, 579

Madau, P. 1995, ApJ, 441, 18

Madau, P., Ferguson, H. C., Dickinson, M. E., Giavalisco, M., Steidel, C. C., \& Fruchter, A. 1996, MNRAS, 283, 1388

Magdziarz, P., \& Zdziarski, A. A. 1995, MNRAS, 273, 837

Magorrian, J., et al. 1998, AJ, 115, 2285

Maiolino, R., Schneider, R., Oliva, E., Bianchi, S., Ferrara, A., Mannucci, F., Pedani, M., \& Roca Sogorb, M. 2004, Nature, 431,533

Marconi, A., et al. 2003, ApJ, 586, 868

Marconi, A., Risaliti, G., Gilli, R., Hunt, L. K., Maiolino, R., \& Salvati, M. 2004, MNRAS, 351, 169

McKee, C. F., \& Ostriker, J. P. 1977, ApJ, 218, 148

Mihos, J. C., \& Hernquist, L. 1994, ApJ, 431, L9

-. 1996, ApJ, 464, 641

Mo, H. J., Mao, S., \& White, S. D. M. 1998, MNRAS, 295, 319

Mobasher, B., et al. 2005, ApJ, 635, 832

Nagamine, K., Cen, R., Furlanetto, S. R., Hernquist, L., Night, C., Ostriker, J. P., \& Ouchi, M. 2006, New Astronomy Review, 50,29

Nagamine, K., Cen, R., Hernquist, L., Ostriker, J. P., \& Springel, V. 2005a, ApJ, 627, 608

-. 2005b, ApJ, 618, 23

Nagamine, K., Springel, V., \& Hernquist, L. 2004, MNRAS, 348, 421

Narayanan, D., et al. 2006, ApJ, Submitted

Night, C., Nagamine, K., Springel, V., \& Hernquist, L. 2006, MNRAS, 366, 705

Noguchi, M. 1988, A\&A, 203, 259

Oke, J. B. 1974, ApJS, 27, 21

Ouchi, M., et al. 2005, ApJ, 620, L1

-. 2004, ApJ, 611, 660

Overzier, R. A., Bouwens, R. J., Illingworth, G. D., \& Franx, M. 2006, ArXiv Astrophysics e-prints

Papovich, C., Dickinson, M., \& Ferguson, H. C. 2001, ApJ, 559, 620

Papovich, C., et al. 2006, ApJ, 640, 92

Pei, Y. C. 1992, ApJ, 395, 130

Porciani, C., Magliocchetti, M., \& Norberg, P. 2004, MNRAS, 355,1010

Porciani, C., \& Norberg, P. 2006, MNRAS, 371, 1824

Power, C., Navarro, J. F., Jenkins, A., Frenk, C. S., White, S. D. M., Springel, V., Stadel, J., \& Quinn, T. 2003, MNRAS, 338, 14

Press, W. H., \& Schechter, P. 1974, ApJ, 187, 425

Reddy, N. A., \& Steidel, C. C. 2004, ApJ, 603, L13

Reddy, N. A., Steidel, C. C., Erb, D. K., Shapley, A. E., \& Pettini, M. 2006, ArXiv Astrophysics e-prints

Réfrégier, A., et al. 2006, in Proc. SPIE Vol. 6265, p. 62651Y, Space Telescopes and Instrumentation I, ed. J. C. Mather, H. A. MacEwen \& M. W. M. de Graauw

Richard, J., Pello, R., Schaerer, D., Le Borgne, J.-F., \& Kneib, J.-P. 2006, ArXiv Astrophysics e-prints

Robertson, B., Cox, T. J., Hernquist, L., Franx, M., Hopkins, P. F., Martini, P., \& Springel, V. 2006a, ApJ, 641, 21

Robertson, B., Hernquist, L., Cox, T. J., Di Matteo, T., Hopkins, P. F., Martini, P., \& Springel, V. 2006b, ApJ, 641, 90

Rodrigues, C. V., Magalhaes, A. M., Coyne, G. V., \& Piirola, V. 1997, ApJ, 485, 618

Salpeter, E. E. 1955, ApJ, 121, 161

Sanders, D. B., \& Mirabel, I. F. 1996, ARA\&A, 34, 749

Sanders, D. B., Soifer, B. T., Elias, J. H., Madore, B. F., Matthews, K., Neugebauer, G., \& Scoville, N. Z. 1988a, ApJ, 325,74

Sanders, D. B., Soifer, B. T., Elias, J. H., Neugebauer, G., \& Matthews, K. 1988b, ApJ, 328, L35

Santos, M. R. 2004, MNRAS, 349, 1137

Schmidt, M. 1959, ApJ, 129, 243

Schneider, D. P., et al. 2001, AJ, 121, 1232

Schneider, D. P., Schmidt, M., \& Gunn, J. E. 1991, AJ, 101, 2004

Shapley, A. E., Steidel, C. C., Erb, D. K., Reddy, N. A., Adelberger, K. L., Pettini, M., Barmby, P., \& Huang, J. 2005, ApJ, 626, 698

Shen, Y., et al. 2007, ArXiv Astrophysics e-prints

Sheth, R. K., \& Tormen, G. 1999, MNRAS, 308, 119

Silk, J., \& Rees, M. J. 1998, A\&A, 331, L1
Skrutskie, M. F., et al. 1997, in ASSL Vol. 210: The Impact of Large Scale Near-IR Sky Surveys, ed. F. Garzon, N. Epchtein, A. Omont, B. Burton, \& P. Persi, 25-+

Soifer, B. T., et al. 1984, ApJ, 278, L71

Soifer, B. T., Sanders, D. B., Madore, B. F., Neugebauer, G., Danielson, G. E., Elias, J. H., Lonsdale, C. J., \& Rice, W. L. 1987, ApJ, 320, 238

Somerville, R. S., Primack, J. R., \& Faber, S. M. 2001, MNRAS, 320,504

Spergel, D. N., et al. 2003, ApJS, 148, 175

Spinrad, H., Stern, D., Bunker, A., Dey, A., Lanzetta, K., Yahil,

A., Pascarelle, S., \& Fernández-Soto, A. 1998, AJ, 116, 2617

Springel, V. 2005, MNRAS, 364, 1105

Springel, V., Di Matteo, T., \& Hernquist, L. 2005a, ApJ, 620, L79

-. 2005b, MNRAS, 361, 776

Springel, V., \& Hernquist, L. 2003, MNRAS, 339, 289

Stanway, E. R., Bunker, A. J., \& McMahon, R. G. 2003, MNRAS, 342, 439

Stanway, E. R., Bunker, A. J., McMahon, R. G., Ellis, R. S., Treu, T., \& McCarthy, P. J. 2004a, ApJ, 607, 704

Stanway, E. R., et al. 2004b, ApJ, 604, L13

Stanway, E. R., McMahon, R. G., \& Bunker, A. J. 2005, MNRAS, 359, 1184

Stark, D. P., Bunker, A. J., Ellis, R. S., Eyles, L. P., \& Lacy, M. 2006, ArXiv Astrophysics e-prints

Steidel, C. C., Pettini, M., \& Hamilton, D. 1995, AJ, 110, 2519

Steidel, C. C., Giavalisco, M., Pettini, M., Dickinson, M., \& Adelberger, K. L. 1996, ApJ, 462, L17+

Steidel, C. C., \& Hamilton, D. 1992, AJ, 104, 941

-. 1993, AJ, 105, 2017

Stern, D., et al. 2005a, ApJ, 631, 163

Stern, D., Yost, S. A., Eckart, M. E., Harrison, F. A., Helfand, D. J., Djorgovski, S. G., Malhotra, S., \& Rhoads, J. E. 2005b, ApJ, 619, 12

Stiavelli, M., Fall, S. M., \& Panagia, N. 2004, ApJ, 610, L1

Strateva, I., et al. 2001, AJ, 122, 1861

Taniguchi, Y., et al. 2005, PASJ, 57, 165

Telfer, R. C., Zheng, W., Kriss, G. A., \& Davidsen, A. F. 2002, ApJ, 565, 773

Thompson, R. I., et al. 2005, AJ, 130, 1

Toomre, A. 1977, in Evolution of Galaxies and Stellar Populations, 401-+

Toomre, A., \& Toomre, J. 1972, ApJ, 178, 623

Tremaine, S., et al. 2002, ApJ, 574, 740

van Dokkum, P. G. 2005, AJ, 130, 2647

van Dokkum, P. G., \& Franx, M. 2001, ApJ, 553, 90

van Dokkum, P. G., et al. 2004, ApJ, 611, 703

Vanden Berk, D. E., et al. 2001, AJ, 122, 549

Vanzella, E., et al. 2006, A\&A, 454, 423

Vázquez, G. A., \& Leitherer, C. 2005, ApJ, 621, 695

Verma, A., Lehnert, M. D., Foerster Schreiber, N. M., Bremer, M. N., \& Douglas, L. 2007, ArXiv Astrophysics e-prints

Vignali, C., Brandt, W. N., \& Schneider, D. P. 2003, AJ, 125, 433

Volonteri, M., Haardt, F., \& Madau, P. 2003, ApJ, 582, 559

Wake, D. A., et al. 2004, ApJ, 610, L85

Walter, F., et al. 2003, Nature, 424, 406

Walter, F., Carilli, C., Bertoldi, F., Menten, K., Cox, P., Lo, K. Y., Fan, X., \& Strauss, M. A. 2004, ApJ, 615, L17

Warren, S., \& Hewett, P. 2002, in ASP Conf. Ser. 283: A New Era in Cosmology, ed. N. Metcalfe \& T. Shanks, 369-+

Warren, S. J., et al. 2007, MNRAS, 375, 213

Warren, S. J., Hewett, P. C., Irwin, M. J., McMahon, R. G., \& Bridgeland, M. T. 1987, Nature, 325, 131

Wechsler, R. H., Bullock, J. S., Primack, J. R., Kravtsov, A. V., \& Dekel, A. 2002, ApJ, 568, 52

Weinberg, D. H., Hernquist, L., \& Katz, N. 2002, ApJ, 571, 15

Werner, M. W., et al. 2004, ApJS, 154, 1

Wiklind, T., Mobasher, B., Dickinson, M., Ferguson, H. C., Giavalisco, M., Grogin, N. A., \& Panagia, N. 2007a, IAU Symposium, 235, 368

Wiklind, T., Dickinson, M., Ferguson, H. C., Giavalisco, M., Mobasher, B., Grogin, N. A., \& Panagia, N. 2007b, ApJ, submitted.

Williams, R. E., et al. 1996, AJ, 112, 1335

Willott, C. J., Rawlings, S., Jarvis, M. J., \& Blundell, K. M. 2003, MNRAS, 339, 173

Wyithe, J. S. B., \& Loeb, A. 2003, ApJ, 595, 614 
Yan, H., et al. 2004, ApJ, 616, 63

Yan, H., Dickinson, M., Giavalisco, M., Stern, D., Eisenhardt, P. R. M., \& Ferguson, H. C. 2006, ApJ, 651, 24

Yan, H., et al. 2005, ApJ, 634, 109

Yan, H., \& Windhorst, R. A. 2004, ApJ, 612, L93

Yan, H., Windhorst, R. A., \& Cohen, S. H. 2003, ApJ, 585, L93

York, D. G., et al. 2000, AJ, 120, 1579

Yoshida, N., Abel, T., Hernquist, L., \& Sugiyama, N. 2003, ApJ, 592,645
Yoshida, N., Omukai, K., Hernquist, L., \& Abel, T. 2006, ApJ, 652,6

Young, J. S., Schloerb, F. P., Kenney, J. D., \& Lord, S. D. 1986, ApJ, 304, 443

Zamorani, G., et al. 1981, ApJ, 245, 357 